\title{
Wind inflow observation from load harmonics
}

\author{
Marta Bertelè $^{1}$, Carlo L. Bottasso ${ }^{1,2}$, Stefano Cacciola ${ }^{2}$, Fabiano Daher Adegas ${ }^{3}$, and Sara Delport ${ }^{3}$ \\ ${ }^{1}$ Wind Energy Institute, Technische Universität München, 85748 Garching bei München, Germany \\ ${ }^{2}$ Dipartimento di Scienze e Tecnologie Aerospaziali, Politecnico di Milano, 20156 Milan, Italy \\ ${ }^{3}$ GE Global Research, 85748 Garching bei München, Germany
}

Correspondence: Carlo L. Bottasso (carlo.bottasso@tum.de)

Received: 16 May 2017 - Discussion started: 20 June 2017

Revised: 23 September 2017 - Accepted: 2 October 2017 - Published: 19 December 2017

\begin{abstract}
The wind field leaves its fingerprint on the rotor response. This fact can be exploited by using the rotor as a sensor: by looking at the rotor response, in the present case in terms of blade loads, one may infer the wind characteristics. This paper describes a wind state observer that estimates four wind parameters, namely the vertical and horizontal shears and the yaw and upflow misalignment angles, from out-of-plane and in-plane blade bending moments. The resulting observer provides on-rotor wind inflow characteristics that can be exploited for wind turbine and wind farm control. The proposed formulation is evaluated through extensive numerical simulations in turbulent and nonturbulent wind conditions using a high-fidelity aeroservoelastic model of a multiMW wind turbine.
\end{abstract}

\section{Introduction}

The wind blowing over a wind turbine rotor leaves its own specific fingerprint on the machine response. If this information is rich enough and if the wind turbine response can be measured (for example in terms of loads), then one may think of turning the rotor into a wind sensor and use it to infer the wind inflow.

Measurements of the rotor inflow during operation are attractive for a number of reasons, as they may find a wide range of applications. For example, information on the wind speed at the rotor disk is typically useful for wind turbine control, as controller behavior is often scheduled as a function of wind speed. In addition, knowledge of the wind direction with respect to the rotor is necessary not only to maximize energy harvesting, but also because operating with excessive misalignment increases loading. Wake redirection strategies (Fleming et al., 2014; Jimenez et al., 2010) deliberately point the rotor away from the wind with the goal of deflecting the wake and reducing its interaction with downstream machines. This is a control strategy that also requires good knowledge of the wind direction in order to be implemented. Upflow can change significantly in complex terrain applications and, if known, it can be used to reduce loading. The presence of an impinging wake, shed from an up- stream wind turbine, may result in high horizontally sheared flow at the rotor disk. Turbulence intensity (TI) and vertical shear may give indications of the characteristics of the atmosphere, which is information that can be used to optimize wind turbine and wind farm control behavior. More generally, by turning each wind turbine into a wind sensor capable of measuring the local inflow characteristics, one may build a more complete picture of the wind flow within a power plant and provide information that may possibly have a variety of uses.

Unfortunately, high-quality information on wind inflow is generally difficult to obtain. Onboard wind turbines, wind speed is typically measured by cup or sonic anemometers, while direction is provided by wind vanes. These sensors invariably suffer from a number of disturbances, such as the presence of the nacelle, blade passing and wake-induced flow deformation. Although most of these effects can be mitigated by the use of calibrated transfer functions, filtering and ad hoc processing of the raw measurements, all these sensors provide only local information at the specific point in the flow where they are installed. For control applications, it is clear that rotor-equivalent information is generally more appropriate than local data because what determines the overall rotor response is what is felt by the whole rotor rather 
than what takes place at a specific point. Additionally, certain wind characteristics can only be defined over a rotor disk and do not have pointwise equivalents, for example shears. Met masts, being equipped with multiple wind sensors away from the rotor, do not suffer from some of these issues. However, the problem of mapping the information from a met mast to the rotor disk of a wind turbine is generally very difficult to solve, and it will clearly always be prone to possibly severe inaccuracies. With lidar (light detection and ranging), laser-based sensing technology is rapidly becoming a game changer, and other remote sensing solutions are also very promising. While their potential is clearly very real and will probably have a deep impact on wind energy technology, these devices are still not in widespread use, mostly because of cost, reliability, availability and lifetime issues.

In this scenario, wind sensing by using the rotor response seems to offer an attractive alternative. In fact, any wind property estimated from the rotor response will be nonlocal and rotor effective in contrast to local sensors. In addition, this approach provides measurements directly at the rotor disk, avoiding the need for mapping flow characteristics from one point to another.

The rotor-effective wind speed estimator (Van der Hooft and Engelen, 2004; Soltani et al., 2013) is one of the first examples of the use of the rotor response for estimating wind characteristics. In this case, the idea is to use the dynamic torque balance equation: based on a map of the aerodynamic torque (or power) of the rotor over the operating envelope of the machine, one may solve this equation in terms of the unknown wind speed, assuming that the other operational parameters (rotor speed, pitch setting, electrical torque) are measured at each instant in time.

This idea was first generalized by Bottasso et al. (2010), who introduced the concept of the rotor as an anemometer. Instead of using the single torque balance, additional equations for the dynamic equilibrium of the machine were used, including the tower and blade degrees of freedom. As multiple equations are now available, multiple wind states can be estimated in addition to wind speed. Although attractive, the need to estimate some wind turbine states resulted in a fairly complicated formulation.

A much simpler approach was developed later in Bottasso and Riboldi (2014), where the idea was not to use the equations of dynamic equilibrium, but rather to consider the steady-state response of the machine. Specifically, the approach exploited the fact that steady wind conditions are associated with a periodic response of the wind turbine. Therefore, a load-wind model was derived linking the harmonics of the blade out- and in-plane bending moments at the rotor frequency (noted $1 \times$ Rev, i.e., once per revolution) with the wind vertical shear and yaw misalignment. A simple blade flapping model was used to derive and justify the structure of the model, while, for accuracy, its actual coefficients were obtained through identification from a higherfidelity aeroservoelastic model of the wind turbine or directly from field tests. A validation of the observer using field data was described in Bottasso and Riboldi (2015) using the Control Advanced Research Turbine (CART3) (Fleming et al., 2011; Bossanyi et al., 2009). The results indicated a significantly higher correlation of the observer results with respect to a met mast, assumed as ground truth, than for the onboard nacelle anemometer and wind vane. Notwithstanding these very promising results, the same study also showed a marked sensitivity of the results on the wind upflow angle, indicating the probable need for a richer description of the wind field.

Following the idea described in Bottasso et al. (2010), an estimator based on a linearized wind turbine model was proposed in Simley and Pao (2014). The formulation used generator speed, fore-aft nacelle acceleration and collective cosine and sine components of the blade out-of-plane bending moments to estimate, with a Kalman filter, the equivalent wind speed together with the linear vertical and horizontal shears. That study demonstrated the performance of the formulation using nonturbulent wind fields that were exactly parameterized by the assumed wind states. However, the effects of unmodeled wind characteristics (for example, turbulence and yaw or upflow misalignments) were not considered.

The concept of the wind turbine as a wind sensor was recently extended to the detection of wake impingement in Bottasso et al. $(2015,2018)$ and Cacciola et al. $(2016 \mathrm{a})$, where loads are used to detect if and where a wake shed by an upstream wind turbine interferes with the rotor.

Motivated by the very promising validation results both in simulations and in the field, the present paper extends and improves the formulation of Bottasso and Riboldi (2014) and Cacciola et al. (2016b) with the goal of addressing some of their weaknesses.

First, extensive numerical experiments have shown that the load-wind model on which the estimator is based must consider at least four wind states instead of two, i.e., the two yaw misalignment and upflow angles as well as the two horizontal and vertical shears. These four states, together with the mean rotor-equivalent speed, represent the lowest-order full approximation of the wind inflow at the rotor disk: the two angles give the orientation of the mean wind vector with the rotor axis, while mean speed and the two shears describe a tilted planar (or mixed linear-exponential, depending on the type of shears considered) inflow. All of these states leave significant signatures in the low-frequency response of the rotor. Therefore, failure to include one of them in the model will invariably create inaccuracies in the others.

Second, the paper shows that the estimators of these four states should be limited to the use of the $1 \times$ Rev response. In fact, although $2 \times$ Rev harmonics are indeed excited by the four states, these same harmonics are also very significantly excited by turbulence, i.e., by higher-order wind states (describing a nonplanar inflow distribution over the rotor disk). As it is not possible to distinguish the part of the $2 \times$ Rev response caused by the four wind states from the part caused by 
turbulence, inclusion of this higher-order response will result in significant pollution of the estimates.

Third, the paper compares both a linear and a nonlinear (quadratic) load-wind model. Both models are scheduled with respect to wind speed in order to account for the different characteristics of a wind turbine in its wind speed operating range. Numerical experiments show that the two are very similar in performance, with a small improvement in accuracy for the nonlinear model over the linear one.

Fourth, experience has shown that angles (yaw misalignment and upflow) are significantly more difficult to estimate than shears. The paper explains the reason for this behavior from two different perspectives. From a mathematical point of view, an a priori analysis based on the singular value decomposition (SVD) demonstrates that angles have a lower level of observability than shears, implying that any small error or perturbation (in the model, in the measurements, in the numerical solution, etc.) will be significantly amplified during the model inversion necessary for the estimation of the wind states. From a physical point of view, this is also easily explained in terms of the sensitivity of angle of attack changes at the blade section to wind state changes. As angles of attack (and hence loads) change less in response to angle changes than to shear changes, angles are harder to estimate than shears when looking at rotor loads.

Finally, the paper demonstrates the performance of the estimator through extensive numerical simulations performed with a high-fidelity aeroservoelastic model of a multi-MW wind turbine. The numerical results illustrate the excellent ability of the proposed formulation to follow rapid fluctuations in shears. The same results also show very interesting behavior of the angle estimators. In fact, although angle estimates are indeed generally polluted by oscillations that depend on turbulence level, their mean errors are significantly low. An analysis that considers the probability distributions of wind speed and turbulence intensity at a given site shows that the expected average inaccuracy of the angle estimates is remarkably low, i.e., less than 1 degree. This means that angles, although apparently oscillatory on short time horizons, can be followed quite precisely in their mean value changes.

The paper is organized according to the following plan. Section 2 presents the formulation of the observer, first introducing load-wind models that relate wind states and blade harmonics, then describing the identification of the model parameters with a system identification approach and finally inverting the model to give wind states when loads are measured. A first set of simulations is used to motivate the limitation of the load vector to the $1 \times$ Rev harmonics. To this end, the simulation environment is briefly introduced together with the aeroservoelastic mathematical model of a wind turbine, which is used throughout the entire work to support all numerical experiments. Section 3 is devoted to an a priori observability analysis of the wind parameters using the SVD followed by a concise summary of the expected observer behavior given in Sect. 3.2. The results of extensive testing of the proposed method in nonturbulent and turbulent wind conditions are given in Sect. 4. Finally, Sect. 5 completes the paper by listing the main conclusions and suggesting possible further improvements to the methodology.

\section{Formulation}

\subsection{Wind anisotropy and its parameterization}

The development of the proposed wind inflow observer is inspired by the idea of using the wind turbine as an anemometer. In this sense, wind is not only the source of energy to be harvested, but also one of the principal factors affecting the wind turbine response. Specifically, the present observer is based on the lowest load harmonics. Although other response indicators could be used in principle, for example accelerations, loads are considered in this work because they are now often measured onboard modern large wind turbines for enabling load feedback control, and load sensors will probably be standard equipment available on most future machines.

In order to understand the connection between blade loads and wind characteristics, consider two different constant-intime wind fields. A first wind field is axially symmetric with respect to the rotation axis of the wind turbine rotor, while the second is not in magnitude or direction. In the second - anisotropic - case, differences in speed and/or direction over the rotor disk may be due to wind shears (both vertical and horizontal) and/or misalignments with the wind direction (due to both yawed flow and upflow caused by rotor uptilt, terrain orography, etc.). In the axially symmetric case, the angle of attack experienced by the blade sections during their azimuthal travel over the rotor disk will be constant; hence, the resulting aerodynamic loads will also be constant. In the non-axially symmetric case, any anisotropy in the wind will cause periodic fluctuations in the angle of attack at the blade sections and hence periodic loads. The amplitude and phase of such loads will depend on the wind field at the rotor disk and on the aeroelastic characteristics of the rotor blades. Therefore, the amplitude and phase of the periodic loads carry information on the wind anisotropy at the rotor disk. This fact can be readily verified with simplified mathematical models of a rotating blade in an anisotropic wind field, for example the classical flapping and lagging blade model developed in Eggleston and Stoddard (1987). Using such a model, Bottasso and Riboldi (2014) suggested a linear structure for a blade-response-based observer of cross-flow and vertical shear.

In this work, the wind field anisotropy is parameterized using four variables (termed wind states in the following): the vertical shear exponent $\kappa_{\mathrm{v}}$, horizontal linear shear $\kappa_{\mathrm{h}}$ and the two angles $\phi$ and $\chi$ measuring the yaw misalignment and upflow, respectively. These quantities are collected in the wind state vector $\boldsymbol{\theta}$, defined as

$\boldsymbol{\theta}=\left(\phi, \kappa_{\mathrm{v}}, \chi, \kappa_{\mathrm{h}}\right)^{T}$ 
More complex wind distributions over the rotor disk might be modeled using higher-order terms. However, such local fluctuations would manifest themselves in higher Rev harmonics, complicating the estimation process.

The wind states are defined with respect to a nacelleattached frame of reference with origin at the hub made of three mutually orthogonal unit vectors $\boldsymbol{x}, \boldsymbol{y}$ and $z$. The $\boldsymbol{x}$ vector is parallel to the rotor axis and pointing downwind and $z$ points upward in the vertical plane, while $\boldsymbol{y}$ is defined according to the right-hand rule. The wind vector $\vec{V}$ is expressed in terms of its components in the nacelle frame as $\boldsymbol{V}=(u, v, w)^{T}$. The wind speed at the rotor disk $W(y, z)=|\boldsymbol{V}|$ is readily computed as

$W(y, z)=V\left(\left(\frac{H+z}{H}\right)^{\kappa_{\mathrm{v}}}+\frac{y}{R} \kappa_{\mathrm{h}}\right)$,

where $V$ is the wind speed at hub height $H$, while $R$ is the rotor radius. The three wind velocity vector components are then expressed as

$$
\begin{aligned}
u(y, z) & =W(y, z) \cos (\phi) \cos (\chi), \\
v(y, z) & =W(y, z) \sin (\phi) \cos (\chi), \\
w(y, z) & =W(y, z) \sin (\chi)
\end{aligned}
$$

Note that because of the definition of the nacelle-attached reference frame $(\boldsymbol{x}, \boldsymbol{y}, \boldsymbol{z})$, a horizontal wind results in an upflow equal to the negative of the nacelle uptilt angle. This is useful for separating the effects of gravitational loads from aerodynamic ones, as shown later. To ease the interpretation of the results, all computed wind states reported in the numerical examples of the rest of this paper were mapped to a frame of reference similar to the nacelle-attached one, but with an $\boldsymbol{x}$ unit vector that is horizontal with respect to the ground instead of being aligned with the rotor axis. Figure 1 illustrates the meaning of the four wind states.

Two different wind fields are considered in the following. In the fully parameterized case, the wind field is completely defined at each instant in time by $V$ and $\boldsymbol{\theta}$. A more realistic wind field is generated using the Kaimal turbulent wind model implemented in the open-source code TurbS im (Jonkman and Kilcher, 2012). In the latter case, the wind field can be considered the superposition of a fully parameterized wind with turbulent fluctuations possessing specific space-time characteristics. Given a wind turbine operating in a turbulent wind field, the goal of the proposed observer is to estimate online a wind state $\boldsymbol{\theta}$ that approximates the turbulent wind at each instant in time.

\subsection{Blade load harmonics}

Under the effects of a steady anisotropic wind, the response of a stable wind turbine converges to a periodic motion. In such a regime, a generic blade load $m$ can be expanded in

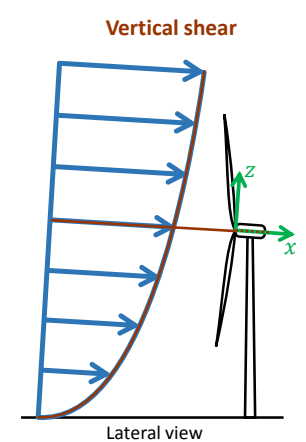

Horizontal shear
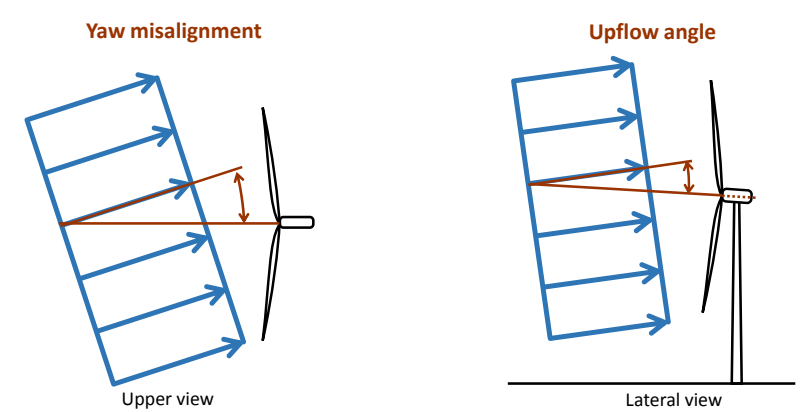

Figure 1. Definition of the four wind states used for parameterizing the wind field over the rotor disk.

Fourier series as

$m(\psi)=m_{0}+\sum_{n=1}^{\infty}\left(m_{n \mathrm{c}} \cos (n \psi)+m_{n \mathrm{~s}} \sin (n \psi)\right)$,

where $\psi$ is the azimuth angle, the subscripts $(\cdot)_{n \mathrm{c}}$ and $(\cdot)_{n \mathrm{~s}}$ refer to the $n \times \operatorname{Rev}$ cosine and sine components, respectively, and $m_{0}$ is the 0th harmonic constant amplitude. The signal harmonics can be computed by demodulating the blade load $m(\psi)$ or, for rotors with at least three blades, by using the Coleman-Feingold (or multi-blade coordinate) transformation (Coleman and Feingold, 1958; Bottasso and Riboldi, 2014). By using the latter method, harmonics at the $n \times \operatorname{Rev}$ frequency can be computed as

$$
\begin{aligned}
\left\{\begin{array}{c}
m_{n \mathrm{c}} \\
m_{n \mathrm{~s}}
\end{array}\right\}= & \frac{2}{3}\left[\begin{array}{ccc}
\cos \left(n \psi_{(1)}\right) & \cos \left(n \psi_{(2)}\right) & \cos \left(n \psi_{(3)}\right) \\
\sin \left(n \psi_{(1)}\right) & \sin \left(n \psi_{(2)}\right) & \sin \left(n \psi_{(3)}\right)
\end{array}\right] \\
& \left\{\begin{array}{c}
m_{(1)} \\
m_{(2)} \\
m_{(3)}
\end{array}\right\}
\end{aligned}
$$

where $m_{(i)}$ and $\psi_{(i)}$ are the $i$ th blade moment and azimuth angle, respectively. Similar relationships also exist for a higher number of blades but not for a smaller number. It can be shown that harmonics at the $i \times \operatorname{Rev}$ are thus transformed into $0 \times \operatorname{Rev}$ components, whereas the other harmonics are either canceled out or transformed into multiples of the number $B$ of blades. This implies that it is always necessary to filter around and above the $B \times \operatorname{Rev}$ frequency after having applied the Coleman transformation. Adaptive filtering can 
be used to follow rotor speed changes caused by variations in the wind speed.

Both out-of-plane (superscript $(\cdot)^{\mathrm{OP}}$ ) and in-plane (superscript $(\cdot)^{\mathrm{IP}}$ ) blade bending harmonic components up to a desired Rev frequency are considered and collected in a vector $\boldsymbol{m}$, defined as

$\boldsymbol{m}=\left(m_{1 \mathrm{c}}{ }^{\mathrm{OP}}, m_{1 \mathrm{~s}}{ }^{\mathrm{OP}}, m_{1 \mathrm{c}}{ }^{\mathrm{IP}}, m_{1 \mathrm{~s}}{ }^{\mathrm{IP}}, m_{2 \mathrm{c}}{ }^{\mathrm{OP}}, m_{2 \mathrm{~s}}{ }^{\mathrm{OP}}, m_{2 \mathrm{c}}{ }^{\mathrm{IP}}, m_{2 \mathrm{~s}}{ }^{\mathrm{IP}}, \ldots\right)^{T}$.

\subsection{Wind state observer}

\subsubsection{Modeling of the load-wind relationship}

The formulation of a wind state observer necessitates a model expressing the dependency of the loads on the wind conditions, in particular of the load harmonics $\boldsymbol{m}$ on the wind state vector $\boldsymbol{\theta}$. To this end, first consider a wind turbine model expressed by a set of nonlinear differential equations together with their output relations:

$\boldsymbol{f}(\boldsymbol{x}, \dot{\boldsymbol{x}}, \boldsymbol{u}(\boldsymbol{\theta}, V, \varrho))=\mathbf{0}$,

$\boldsymbol{y}=\boldsymbol{g}(\boldsymbol{x}, \dot{\boldsymbol{x}}, \boldsymbol{u}(\boldsymbol{\theta}, V, \varrho))$,

where $\boldsymbol{x}$ is the state vector, $\boldsymbol{u}$ is the input vector and $\boldsymbol{y}=$ $\left(m^{\mathrm{OP}}, m^{\mathrm{IP}}\right)^{T}$ indicates the output vector (in this case, containing the out- and in-plane components of the blade bending moment $\mathrm{m}$ ). The input vector only includes the exogenous disturbance represented by the wind parameters $\boldsymbol{\theta}$, the wind speed $V$ and the air density $\varrho$ because the presence of a feedback controller (usually in the form of a pitch-torque controller) can be considered to be included in the definition of the system model $f(\cdot)$.

Under a steady input $\boldsymbol{u}$, the response of the system (Eq. 7a) in terms of its states converges to a periodic solution, which can be described through a truncated Fourier expansion as

$\boldsymbol{x}=\boldsymbol{x}_{0}+\sum_{n=1}^{N}\left(\boldsymbol{x}_{n \mathrm{c}} \cos (n \psi)+\boldsymbol{x}_{n \mathrm{~s}} \sin (n \psi)\right)$.

By inserting Eq. (8) into Eq. (7a) and collecting all terms at the same frequency (a procedure termed harmonic balance), one can compute $\boldsymbol{x}_{n \mathrm{c}}$ and $\boldsymbol{x}_{n \mathrm{~s}}$, which will clearly depend on $\boldsymbol{\theta}, V$ and $\varrho$. Finally, the harmonics $\boldsymbol{x}_{n \mathrm{c}}$ and $\boldsymbol{x}_{n \mathrm{~s}}$ can be inserted into the output Eq. (7b), yielding the desired relationship between load harmonics and wind parameters:

$\boldsymbol{m}=\mathcal{M}(\boldsymbol{\theta}, V, \varrho)$.

An example of this derivation for a simplified flapping blade model can be found in Bottasso and Riboldi (2014). In principle, the resulting input-output relationship should also include the dependency on other parameters, such as blade pitch and rotor speed, as shown for example in Simley and Pao (2014). However, all these quantities depend in turn on the environmental and operating conditions according to the particular regulation strategy adopted by the onboard controller. Therefore, in this work the model is assumed to depend only on $\boldsymbol{\theta}, V$ and $\varrho$. Vector $\boldsymbol{\theta}$ is to be estimated with the proposed observer, while $V$, which is a scheduling parameter for the model, can either be measured or observed using a rotor-equivalent wind speed estimator (Soltani et al., 2013; Simley and Pao, 2014; Bottasso et al., 2015, 2018).

This approach leads to a white box model, i.e., a model using analytical formulas to express relationships among the relevant variables based on physical principles (Ljung, 2010). The use of white box models may suffer from inaccuracies. Any mismatch between model (Eq. 7) and reality will inevitably pollute the input-output relationship (Eq. 9). To address this problem, one may calibrate some of the parameters of the model (Eq. 7) based on available measurements. This procedure is carried out using parameter identification techniques (Jategaonkar, 2015) and leads to a gray box model, defined as a white box model in which some parameters are taken as unknown (Ljung, 2010).

In this paper, a third approach is used, which is entirely based on system identification. In this case, the desired input-output relationship between loads and wind states is considered as a black box (Ljung, 2010). In this case, the model does not contain any physics-based formulas, but it is designed to be capable of parameterizing the desired functional dependency in a general and flexible way. This is typically done through a set of assumed bases and associated free parameters (Ljung, 2010), which are then identified directly from measurements of $\boldsymbol{m}$ and $\boldsymbol{\theta}$. This way, the need for an analytical model is bypassed completely. The advantage of avoiding the use of a white or gray model is paid in terms of the need for a set of measurements that is rich and complete enough to enable the identification of the relationship of interest. Also note that although we use a black box approach, which by definition is blind to the physics, we bring specific knowledge on the nature of the problem through the definition of the output vector in terms of load harmonics.

The data set for the identification of the black box model can be obtained either by simulation or by measurements performed in the field. The former approach, which is also the one that was used for the present work, is relatively simple because in a simulation environment one can readily measure all necessary quantities (loads and wind states). In contrast to this simplicity, it is clear that any mismatch between the simulation model and reality will affect the quality of the identified input-output model. While this is in principle a possible drawback, one should not forget that the present approach only uses the very lowest harmonics (typically only the $1 \times$ Rev) of the response. State-of-the-art aeroservoelastic codes used for the design and certification of wind turbines are typically quite accurate in this frequency range. An additional remark on this modeling approach is in order: it is clear that identifying a black box model based on the outputs of a simulation is in a sense akin to the extraction of a white box model from the simulation model itself. However, given the level of complexity of modern comprehensive aeroservoelastic codes, the direct extraction of the necessary inputoutput relationship through the manipulation of the underly- 
ing equations is hardly feasible in practice, especially when working with legacy codes.

Another possible approach is to use field measurements. In this case the machine should be equipped with load sensors, a met mast and a lidar or other flow sensors to measure wind states. Each of these techniques implies its own hypothesis (e.g., frozen turbulence in the case of flow measurements performed away from the rotor disk), each is limited by its own specific inherent accuracy and each is affected by errors and disturbances. While this approach is certainly possible and was in fact successfully demonstrated in Bottasso and Riboldi (2014), it was not pursued further in the present work.

\subsubsection{Linear model}

Inspired by Eq. (9), a linear input-output model can be expressed as

$$
\begin{aligned}
\boldsymbol{m} & =\mathbf{F}(V, \varrho) \boldsymbol{\theta}+\boldsymbol{m}_{0}(V, \varrho), \\
& =\mathbf{T} \overline{\boldsymbol{\theta}},
\end{aligned}
$$

where $\mathbf{F}$ and $\boldsymbol{m}_{0}$ are the model coefficients, while $\mathbf{T}(V, \varrho)=$ $\left[\mathbf{F}(V, \varrho), \boldsymbol{m}_{0}(V, \varrho)\right]$ and $\overline{\boldsymbol{\theta}}=\left(\boldsymbol{\theta}^{T}, 1\right)^{T}$.

Matrix $\mathbf{F}$ is the sensitivity of the harmonics with respect to the wind states and depends on the operating condition of the machine through the wind speed $V$ and the air density $\varrho$. Vector $\boldsymbol{m}_{0}$ is a term accounting for gravity-induced loads. In fact, when $\boldsymbol{\theta}=0$, the wind field is a constant-over-the-rotor-disk flow parallel to the rotor axis, which only causes a $0 \times \operatorname{Rev}$ load response and therefore does not contribute to $\boldsymbol{m}$. Similarly, inertial effects due to the rotor spinning with an angular velocity $\Omega$ also generate only $0 \times$ Rev loads, and hence do not contribute to Eq. (10). Vector $\boldsymbol{m}_{0}$ can be expressed as

$\boldsymbol{m}_{0}=\boldsymbol{g}+q A \boldsymbol{c}(V, \varrho)$.

The first term, $g$, accounts for in-plane and out-of-plane gravity-induced loads, the latter being caused by blade precone, prebend and rotor uptilt. The second term, $q A \boldsymbol{c}$, is a gravity-induced load due to the rotor deformation caused by aerodynamic loads, which can therefore be nondimensionalized accordingly. For the same reasons noted above, this term also generally depends on $V$ and $\varrho$.

Separating the effects of gravity from aerodynamicinduced loads allows for the correction of air density changes. This is important in practice because density, being dependent on temperature, undergoes significant fluctuations in the field, thereby affecting load measurements. The split of gravity-induced terms into constant and aerodynamically caused terms is also important, as it highlights the variability of the latter term with the operating condition.

The unknown matrix of coefficients $\mathbf{T}$ can be computed by collecting multiple observations for the moments $\boldsymbol{m}^{(i)}$ and inputs $\overline{\boldsymbol{\theta}}^{(i)}$, where (.) $)^{(i)}$ indicates the $i$ th of $N_{\text {exp }}$ available ob- servations. By grouping the measurements into the matrices

$\mathbf{M}=\left[\boldsymbol{m}^{(1)}, \boldsymbol{m}^{(2)}, \ldots, \boldsymbol{m}^{\left(N_{\exp }\right)}\right]$,

$\boldsymbol{\Theta}=\left[\overline{\boldsymbol{\theta}}^{(1)}, \overline{\boldsymbol{\theta}}^{(2)}, \ldots, \overline{\boldsymbol{\theta}}^{\left(N_{\mathrm{exp}}\right)}\right]$,

the input-output relationship (10) can be written collectively for all observations as

$\mathbf{M}=\mathbf{T} \Theta$.

Finally, matrix $\mathbf{T}$ is readily estimated in a least-squares sense as

$\mathbf{T}=\mathbf{M} \Theta^{T}\left(\Theta \Theta^{T}\right)^{-1}$.

The problem is solvable if and only if matrix $\Theta$ has a full rank. In this sense, the condition number of matrix $\boldsymbol{\Theta} \Theta^{T}$ gives an indication of the identifiability of a model given a set of measurements. If the condition number is excessively high, then the problem is ill posed and the data set has to be enriched and/or modified.

As previously noted, the input-output model should be scheduled in terms of the wind speed $V$ and air density $\varrho$, as the model coefficients depend on the operating condition of the machine. To this end, a piecewise linear scheduled model can be expressed as

$$
\begin{aligned}
\boldsymbol{m} & =\sum_{k=1}^{N_{\text {node }_{V}}} \sum_{w=1}^{N_{\text {node }}} \mathbf{F}_{k, w} n_{k, w}(V, \varrho) \boldsymbol{\theta}+\boldsymbol{m}_{0 k, w} n_{k, w}(V, \varrho) \\
& =\sum_{k=1}^{N_{\text {node }}} \sum_{w=1}^{N_{\text {node }}} \mathbf{T}_{k, w} n_{k, w}(V, \varrho) \overline{\boldsymbol{\theta}},
\end{aligned}
$$

where the wind speed and air density ranges have been discretized by introducing $N_{\text {node }_{V}}$ wind speed nodes and $N_{\text {node }}$ density nodes, while $\mathbf{F}_{k, w}$ and $\boldsymbol{m}_{0 k, w}$ are the model coefficient nodal matrices grouped together as $\mathbf{T}_{k, w}=$ $\left[\mathbf{F}_{k, w} \boldsymbol{m}_{0 k, w}\right]$. Finally, two-dimensional shape functions are noted $n_{k, w}(V, \varrho)$. The scheduled model (Eq. 15) can be written in a more compact form as

$\boldsymbol{m}=\widehat{\mathbf{T}} \widehat{\boldsymbol{\theta}}(V, \varrho)$

where $\widehat{\boldsymbol{\theta}}(V, \varrho)=\widehat{\mathbf{N}}(V, \varrho) \overline{\boldsymbol{\theta}}$ and

$$
\begin{aligned}
\widehat{\mathbf{T}}= & {\left[\mathbf{T}_{1,1}, \mathbf{T}_{1,2}, \ldots, \mathbf{T}_{k, w}, \ldots, \mathbf{T}_{N_{\text {node }_{V}}, N_{\text {node }}}\right], } \\
\widehat{\mathbf{N}}= & {\left[n_{1,1}(V, \varrho) \mathbf{I}, n_{1,2}(V, \varrho) \mathbf{I}, \ldots, n_{k, w}(V, \varrho) \mathbf{I},\right.} \\
& \left.\ldots, n_{N_{\text {node }_{V}}, N_{\text {node }}(V, \varrho)} \mathbf{I}\right]^{T},
\end{aligned}
$$

where $\mathbf{I}$ is an identity matrix of suitable dimensions.

Samples of the wind states and associated loads are now collected at $N_{\text {exp }}$ different operating conditions, each corresponding to its own wind speed $V^{(i)}$ and air density $\varrho^{(i)}$. The 

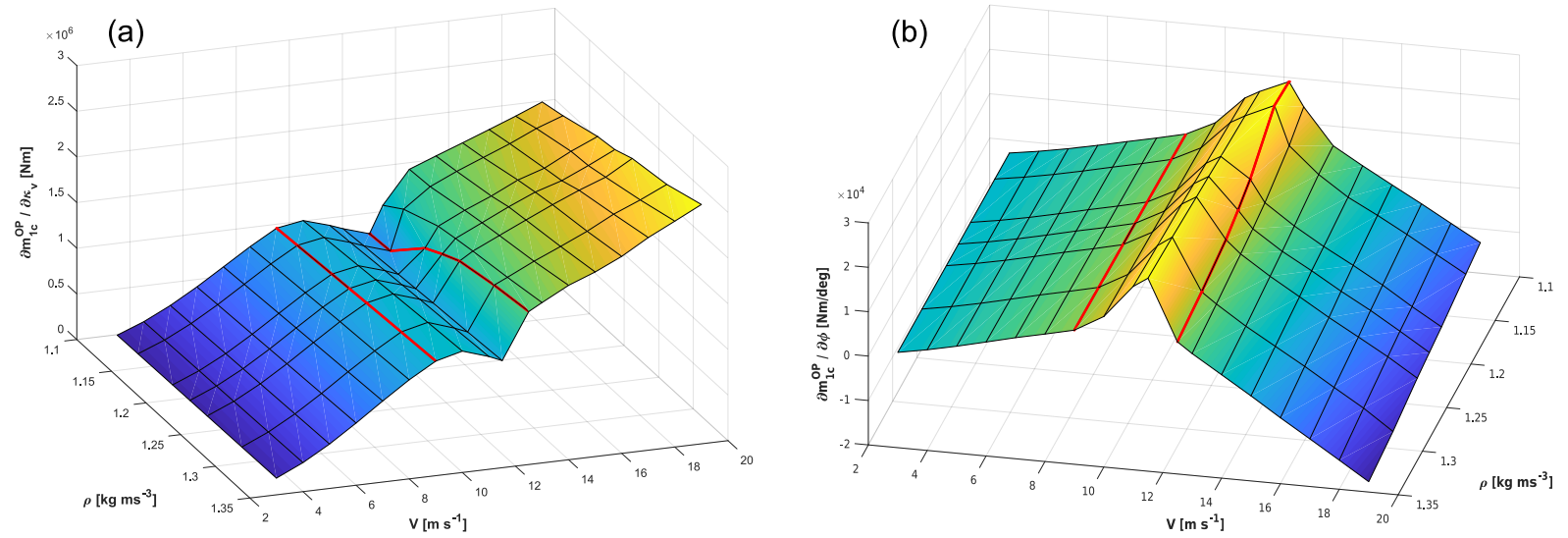

Figure 2. Behavior of two load sensitivities as functions of wind speed $V$ and air density $\varrho$ : sensitivity of out-of-plane $1 \times$ Rev cosine moment with respect to vertical shear $\kappa_{\mathrm{V}}$ (a) and yaw misalignment $\phi(\mathbf{b})$. The boundaries between regions II, II $\frac{1}{2}$ and III are indicated by red solid lines. The color indicates the value of the function, i.e., the elevation of the plot.

$i$ th load vector and wind state vector are notated as $\boldsymbol{m}^{(i)}$ and $\widehat{\boldsymbol{\theta}}\left(V^{(i)}, \varrho^{(i)}\right)$, respectively. Both loads and wind states are collected into matrices as

$$
\begin{aligned}
\widehat{\mathbf{M}}= & {\left[\boldsymbol{m}^{(1)}, \boldsymbol{m}^{(2)}, \ldots, \boldsymbol{m}^{\left(N_{\exp }\right)}\right], } \\
\widehat{\boldsymbol{\Theta}}= & {\left[\widehat{\boldsymbol{\theta}}\left(V^{(1)}, \varrho^{(1)}\right), \widehat{\boldsymbol{\theta}}\left(V^{(2)}, \varrho^{(2)}\right), \ldots,\right.} \\
& \left.\widehat{\boldsymbol{\theta}}\left(V^{\left(N_{\exp }\right)}, \varrho^{\left(N_{\exp }\right)}\right)\right],
\end{aligned}
$$

leading to the overall system

$$
\widehat{\mathbf{M}}=\widehat{\mathbf{T}} \widehat{\boldsymbol{\Theta}} \text {. }
$$

Finally, the matrix of unknown coefficients $\widehat{\mathbf{T}}$ is computed in a least-squares sense as

$$
\widehat{\mathbf{T}}=\widehat{\mathbf{M}} \widehat{\Theta}^{T}\left(\widehat{\boldsymbol{\Theta}} \widehat{\boldsymbol{\Theta}}^{T}\right)^{-1}
$$

The problem is well posed if the solving least-squares matrix, $\widehat{\boldsymbol{\Theta}} \widehat{\boldsymbol{\Theta}}^{T}$, is non-singular. One must clearly ensure that samples adequately cover all wind speed intervals in order to ensure the identifiability of all nodal matrices $\mathbf{F}_{k, w}$ and $\boldsymbol{m}_{0 k, w}$.

An example of the typical behavior of the model coefficients is given in Fig. 2 for the wind turbine described later in this paper. The figure reports $\partial m_{1 \mathrm{c}} \mathrm{OP} / \partial \kappa_{\mathrm{v}}$ (Fig. 2a) and $\partial m_{1 \mathrm{c}} \mathrm{OP} / \partial \phi$ (Fig. 2b) as functions of $V$ and $\varrho$. There is distinctly different behavior with respect to the wind speed of the load sensitivities in regions II (partial load) and III (full load). The rapid changes in the transition region $\mathrm{II} \frac{1}{2}$ call for a suitable refinement of the node spacing in this regime. In general, the situation with respect to density is simpler, with small departures from linear behavior only in the transition region.

\subsubsection{Nonlinear model}

The assumption of linearity in the input-output relationship (Eq. 9) might lead to inaccuracies. To correct for these potential effects while limiting model complexity, a model with an assumed degree of nonlinearity is formulated as

$\boldsymbol{m}=\mathbf{F}_{\mathrm{NL}} \boldsymbol{\theta}_{\mathrm{NL}}+\boldsymbol{m}_{\mathrm{NL}_{0}}$.

The nonlinear wind state vector $\boldsymbol{\theta}_{\mathrm{NL}}$ contains, in addition to the elements of $\boldsymbol{\theta}$, their nonlinear combinations $\theta_{\mathrm{NL}_{j}}$ up to a given order $p$, where

$\theta_{\mathrm{NL} j}=\prod_{i} \theta_{i} \alpha_{i} \quad$ s.t. $\quad \sum_{i} \alpha_{i} \leq p ;$

$\theta_{i}$ is the $i$ th element of the linear wind state vector $\boldsymbol{\theta}$. For $p=2$, which is the case considered here, the nonlinear wind state vector contains 14 terms:

$$
\begin{aligned}
\boldsymbol{\theta}_{\mathrm{NL}}= & \left(\phi, \kappa_{\mathrm{v}}, \chi, \kappa_{\mathrm{h}}, \phi \kappa_{\mathrm{v}}, \phi \chi, \phi \kappa_{\mathrm{h}},\right. \\
& \left.\kappa_{\mathrm{v}} \chi, \kappa_{\mathrm{v}} \kappa_{\mathrm{h}}, \chi \kappa_{\mathrm{h}}, \phi^{2}, \kappa_{\mathrm{v}}{ }^{2}, \chi^{2}, \kappa_{\mathrm{h}}^{2}\right)^{T} .
\end{aligned}
$$

As the nonlinear model (Eq. 21) is linear in the unknown coefficients $\mathbf{F}_{\mathrm{NL}}$ and $\boldsymbol{m}_{\mathrm{NL}_{0}}$, its identification is formally identical to that of the linear model for both the unscheduled and the scheduled cases. However, as more coefficients are present, one has to check that the data set is complete enough to guarantee that the problem is well posed.

\subsubsection{Wind turbine simulation model}

In this work, an aeroservoelastic simulation model is used to represent the dynamic behavior of a wind turbine in all different scenarios of interest. The model represents a horizontal axis wind turbine with a rotor of $93 \mathrm{~m}$ diameter with an uptilt of $4.5^{\circ}$, a hub height of $80 \mathrm{~m}$ and a rated power of $3 \mathrm{MW}$. The 

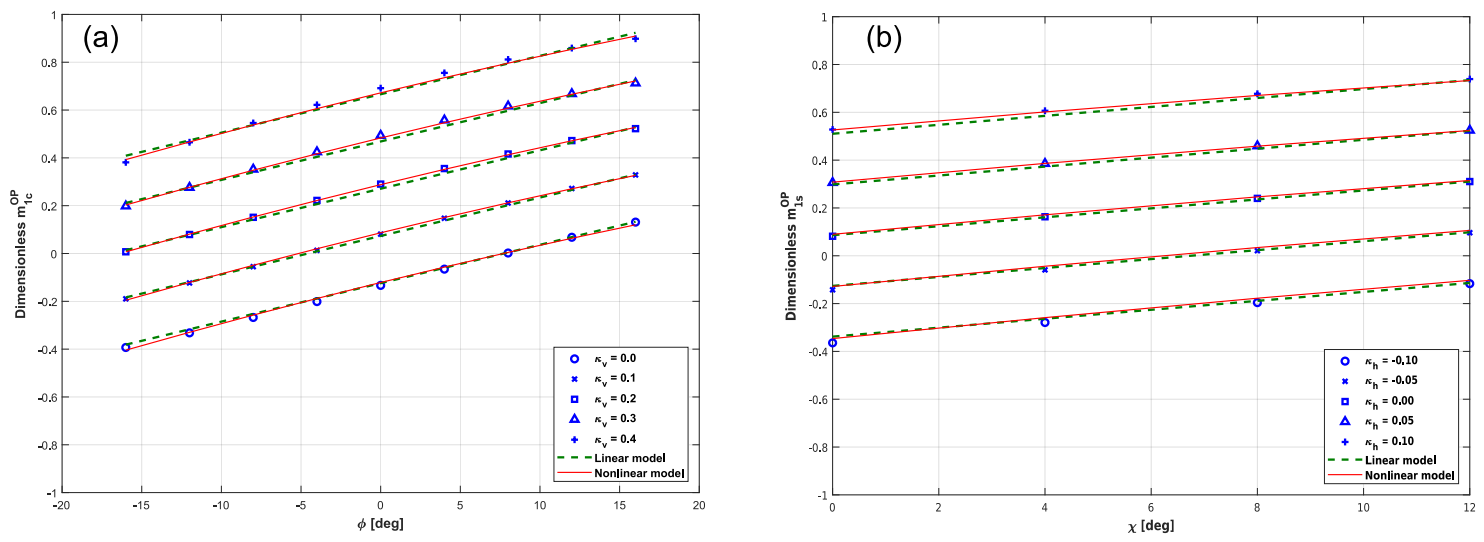

Figure 3. Comparison between measured and predicted harmonics for the linear model (dashed thick lines) and the nonlinear model of order 2 (solid thin lines). Normalized $1 \times$ Rev cosine (a) and sine (b) out-of-plane moment components are shown for different wind state variables.

wind speeds at cut-in $\left(V_{\mathrm{CI}}\right)$, rated power $\left(V_{\mathrm{RP}}\right)$ and cut-out $\left(V_{\mathrm{CO}}\right)$ are respectively equal to $3,12.5$ and $25 \mathrm{~m} \mathrm{~s}^{-1}$. A rather wide transition region $\mathrm{II} \frac{1}{2}$ extends from 9 to $12.5 \mathrm{~m} \mathrm{~s}^{-1}$. The cut-in rotor speed is equal to 5.2 RPM, whereas the rated rotor speed is equal to $15 \mathrm{RPM}$. Both side-side and fore-aft tower frequencies $f_{\text {tower }}$ are equal to $0.3 \mathrm{~Hz}$. The first blade flapwise frequency $f_{\text {flap }}$ varies between $0.9 \mathrm{~Hz}$ at cut-in and $1 \mathrm{~Hz}$ at rated rotor speed. Finally, the first blade edgewise mode $f_{\text {edge }}$ is at around $1.5 \mathrm{~Hz}$.

The aeroservoelastic model of the machine is developed using the finite-element multi-body code $\mathrm{Cp}$-Lambda (Bauchau et al., 2003; Bottasso and Croce, 2006). The model includes flexible blades, a tower and a drivetrain implemented with geometrically exact nonlinear beam models (Bauchau, 2011). Rotor-speed-dependent mechanical losses are considered within the drivetrain generator model, and compliant foundations are used to connect the tower base to the ground. The aerodynamics are rendered through the classical blade element momentum theory (BEM) and consider hub and tip losses, dynamic stall and unsteady corrections. The model is completed by an active pitch torque controller implemented as a speed-scheduled linear quadratic regulator (LQR) (Bottasso et al., 2012; Riboldi, 2012). Additionally, the pitch and torque actuators are modeled as second- and first-order systems, respectively. Finally, the model is subjected to wind time histories generated by the code TurbS im (Jonkman and Kilcher, 2012).

\subsubsection{Load-wind relationship in steady conditions}

To test the performance of the linear and nonlinear models, the wind turbine was simulated in a variety of different operating conditions. Fully parameterized steady winds were generated at $N_{\text {node }_{V}}=10$ speeds $V=$ $\{3,4,5,6,7,8,9,11,15,19\} \mathrm{m} \mathrm{s}^{-1}$, where for each different wind speed all 900 possible combinations of the following wind parameters were considered:

$$
\begin{aligned}
\phi & =\{-16,-12,-8,-4,0,4,8,12,16\} \mathrm{deg}, \\
\kappa_{\mathrm{v}} & =\{0.0,0.1,0.2,0.3,0.4\}, \\
\chi & =\{0,4,8,12\} \mathrm{deg}, \\
\kappa_{\mathrm{h}} & =\{-0.1,-0.05,0.0,0.05,0.1\},
\end{aligned}
$$

resulting in $N_{\exp }=9000$ available observations. Loads were measured on the aeroelastic model in a blade-attached reference frame located at the root of each blade, thereby simulating the presence of strain gages measuring flapwise and edgewise bending moment components, which were then transformed into out- and in-plane rotor components by using the blade pitch angle. Next, the out- and in-plane loads were decomposed into their harmonics at the $1 \times \operatorname{Rev}$ and $2 \times \operatorname{Rev}$ with the Coleman transformation and used together with the corresponding wind states to identify the linear and nonlinear models used throughout this work.

From the full range of tests performed, Fig. 3 shows two representative examples at a wind speed of $7 \mathrm{~m} \mathrm{~s}^{-1}$, illustrating the match between the measurements obtained on the wind turbine simulation model (taken as ground truth) and the outputs of the identified models. The ground truth is reported with markers, the linear model with dashed lines and the nonlinear model with solid lines. Figure 3a shows $m_{1 \mathrm{c}}$ OP as a function of $\phi$ for different values of $\kappa_{\mathrm{v}}$ and for $\kappa_{\mathrm{h}}=0.0$ and $\chi=4^{\circ}$. Figure $3 \mathrm{~b}$ shows $m_{1 \mathrm{~s}} \mathrm{OP}$ as a function of $\chi$ for different values of $\kappa_{\mathrm{h}}$ and for $\kappa_{\mathrm{v}}=0.0$ and $\phi=0^{\circ}$. Both moments are nondimensionalized with respect to their own maximum absolute values.

The figure shows that both models are capable of capturing the relevant behavior of the harmonics with respect to wind states. The relationships appear to be linear, with only very minor nonlinearities. These analyses also graphically illustrate the sensitivity of the loads with respect to the wind parameters. As expected, even though all parameters have a certain effect on all loads, cosine harmonics are mainly influenced by the couple $\left\{\phi, \kappa_{\mathrm{v}}\right\}$, whereas sine harmonics are 

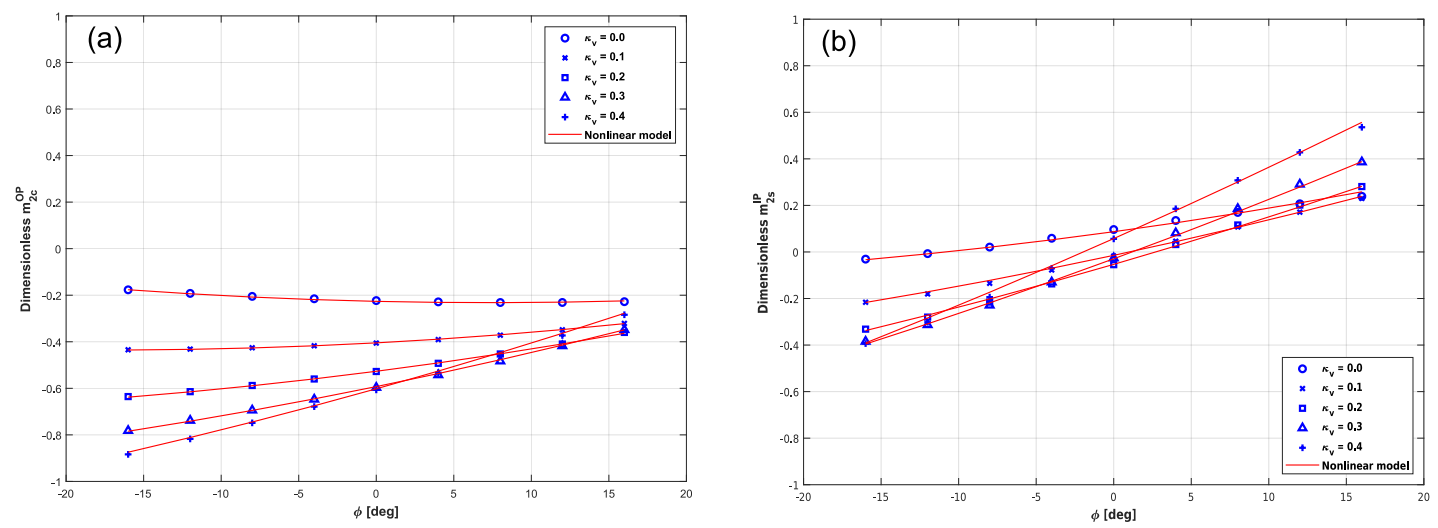

Figure 4. Comparison between measured and predicted harmonics for the nonlinear model of order 2 (solid thin lines). Normalized out-ofplane $2 \times$ Rev cosine (a) and in-plane $2 \times \operatorname{Rev}$ sine (b) are shown for different wind state variables.

influenced by $\left\{\chi, \kappa_{\mathrm{h}}\right\}$. Similar considerations can be derived for the in-plane harmonics, which are not shown here for the sake of brevity.

In contrast, the $2 \times \operatorname{Rev}$ harmonics have markedly different behavior, as shown in Fig. 4. The plots report the nondimensional out-of-plane $2 \times$ Rev cosine term (Fig. 4a) and the in-plane $2 \times$ Rev sine term (Fig. 4b) as functions of $\phi$ and for varying $\kappa_{\mathrm{v}}$, with $\kappa_{\mathrm{h}}=0.0$ and $\chi=4^{\circ}$. Given the clear nonlinearity of the relationships, only the nonlinear model is able to capture the correct trends of these higher harmonics with respect to the wind states.

\subsubsection{Choosing the number of harmonics}

The previous analysis performed in steady wind conditions showed that the $1 \times$ Rev harmonics exhibit largely linear behavior with respect to the wind states, while the $2 \times$ Revs exhibit marked nonlinearities. In order to understand the behavior of the models in more realistic conditions, simulations were conducted in turbulent winds. In particular, it is necessary to establish whether the unsteadiness in the excitation provided by a turbulent wind is compatible with the steady-state harmonic models considered herein. In addition, as previously noted, a turbulent wind field cannot generally be exactly represented by the reduced set of wind states.

To investigate these effects, a 10 min simulation was performed at a $5 \mathrm{~m} \mathrm{~s}^{-1}$ mean wind speed with a TI equal to $20 \%$ and null mean yaw misalignment, upflow and vertical and horizontal shears. At each instant in time, values of the wind parameters were computed from the wind grid generated with Turbsim (Jonkman and Kilcher, 2012) by fitting in a least-squares sense Eqs. (2) and (3). Blade load harmonics were extracted from the simulated outputs using the Coleman transformation and filtered with a low-pass sixth-order Butterworth filter with a cut-out frequency of $0.14 \mathrm{~Hz}$ in order to remove the remaining $3 \times$ Rev harmonic content in the Coleman-transformed moments. Figure 5 shows a comparison of the harmonics extracted from the simulation (shown as a thick blue solid line and again assumed to represent the ground truth) with those predicted by the second-order nonlinear model (shown as a thin red solid line) fed with the wind parameters computed from the wind grid. Figure 5a shows moment $m_{1 \mathrm{c}}{ }^{\mathrm{IP}}$, while Fig. $5 \mathrm{~b}$ shows moment $m_{2 \mathrm{c}}$ OP.

By looking at Fig. 5a, it appears that there is an excellent match between the predictions and measurements for the inplane $1 \times \operatorname{Rev}$ cosine harmonic. The small delay between the two signals is due to the filter used for removing higher frequencies. Both the linear and nonlinear models yield similarly accurate results for the sine and out-of-plane components, which are not reported here for brevity. These results show that $1 \times$ Rev harmonics are primarily influenced by the wind states used here for parameterizing the wind field, with only small disturbances caused by turbulent fluctuations and blade dynamic effects. In this sense, $1 \times$ Rev harmonics are good candidates for feeding a wind state observer.

In contrast, Fig. 5b shows completely different behavior of the measurements and predictions for the $2 \times$ Rev components in turbulent conditions. It should be remarked that, as previously illustrated in Fig. 4, the model is perfectly capable of capturing these higher harmonics with good accuracy in steady wind conditions. The reason for the very poor results in the turbulent case is that small-scale turbulent fluctuations in the wind field cause $2 \times \operatorname{Rev}$ harmonics that are comparable to, if not larger than, those caused by the wind states used for the parameterization. Therefore, although $2 \times \operatorname{Rev}$ harmonics carry information on the wind states, this information cannot be separated from the pollution brought by the smaller-scale wind field fluctuations. In this sense, $2 \times \operatorname{Rev}$ harmonics are not good candidates for the observation of wind states. Based on these results, the vector of blade harmonics is limited in the rest of this work to $1 \times$ Revs and simply defined as

$\boldsymbol{m}=\left(m_{1 \mathrm{c}}^{\mathrm{OP}}, m_{1 \mathrm{~s}}^{\mathrm{OP}}, m_{1 \mathrm{c}}^{\mathrm{IP}}, m_{1 \mathrm{~s}}^{\mathrm{IP}}\right)^{T}$. 

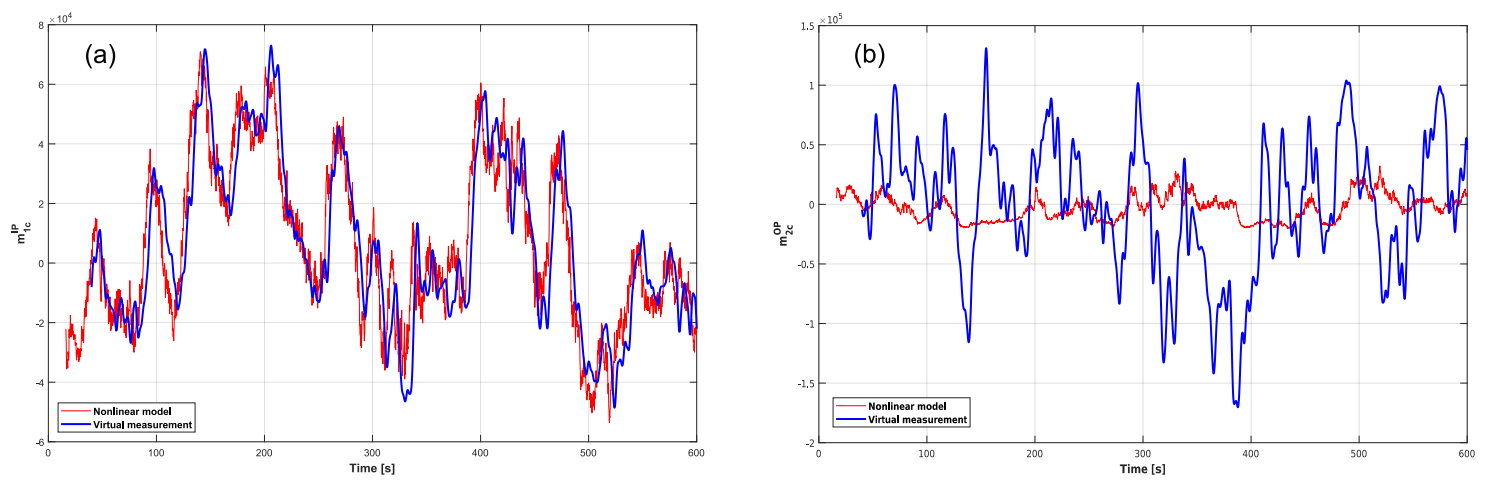

Figure 5. Comparison in turbulent wind conditions between measured harmonics (thick solid line) and harmonics predicted with a secondorder nonlinear model (thin solid line). (a) In-plane $1 \times$ Rev cosine component; (b) out-of-plane $2 \times$ Rev cosine component.

\subsubsection{Wind state estimation}

The problem of computing an estimate $\boldsymbol{\theta}_{\mathbf{E}}$ of the wind state vector given a load harmonic vector $\boldsymbol{m}_{\mathrm{M}}$ is considered next. Given the input-output model (Eq. 9), a measured load $\boldsymbol{m}_{\mathrm{M}}$ can be expressed as

$\boldsymbol{m}_{\mathrm{M}}=\mathcal{M}(\boldsymbol{\theta}, V, \varrho)+\boldsymbol{r}$,

where $\boldsymbol{r}$ is the measurement error with covariance $\mathbf{R}=$ $\mathbf{E}\left[\boldsymbol{r} \boldsymbol{r}^{T}\right]$. The residual is assumed to be zero-mean, white and Gaussian. The residual is due not only to measurement noise, but also to all effects not captured by the model, such as sampling and discretization errors, unmodeled nonlinearities and turbulence-induced loads. This implies that the assumption of a zero-mean, white and Gaussian noise might be far from real.

The generalized least-squares estimate of $\boldsymbol{\theta}$ given $\boldsymbol{m}_{\mathrm{M}}$ is

$$
\begin{aligned}
\boldsymbol{\theta}_{\mathbf{E}}= & \arg \min _{\theta} \\
& \left(\left(\boldsymbol{m}_{\mathrm{M}}-\mathcal{M}(\boldsymbol{\theta}, V, \varrho)\right)^{T} \mathbf{R}^{-1}\left(\boldsymbol{m}_{\mathrm{M}}-\mathcal{M}(\boldsymbol{\theta}, V, \varrho)\right)\right) .
\end{aligned}
$$

Consider the linear model (Eq. 10a) and assume $V$ to be known. The solution to the problem in Eq. (27) can be worked out analytically as

$\boldsymbol{\theta}_{\mathbf{E}}=\left(\mathbf{F}(V)^{T} \mathbf{R}^{-1} \mathbf{F}(V)\right)^{-1} \mathbf{F}(V)^{T} \mathbf{R}^{-1}\left(\boldsymbol{m}_{\mathrm{M}}-\boldsymbol{m}_{0}\right)$.

Vector $\boldsymbol{\theta}_{\mathbf{E}}$ is structurally identifiable (or observable) if matrix $\mathbf{F}(V)^{T} \mathbf{R}^{-1} \mathbf{F}(V)$ is non-singular. The structural identifiability analysis, which reveals when the estimation problem is well posed and to what degree of accuracy it can be solved, will be analyzed in Sect. 3.

For the nonlinear model (Eq. 21), the solution to the problem (Eq. 27) involves a nonlinear unconstrained minimization, which was solved here starting from a suitable initial guess by using the Levenberg-Marquardt method (More, 1977). As multiple local solutions may characterize the nonlinear problem, a global search algorithm or multiple starting points should be used to find the optimum. Here again, one must verify observability, as discussed later in Sect. 3.

The estimator (Eq. 27) was first characterized in steady wind conditions, and the results of this analysis are shown next. All plots are arranged in a similar way: any estimated wind state variable is plotted on the $y$ axis as a function of its corresponding ground truth quantity, which is reported on the $x$ axis. A black thin solid line indicates the bisector of the plot, representing a perfect match between the two quantities. Estimates are plotted using markers and thick solid lines for different wind conditions. Clearly, any deviation from the bisector directly indicates an estimation error.

Figure 6 shows an excerpt of the results obtained with the linear model for different wind conditions at $5 \mathrm{~m} \mathrm{~s}^{-1}$. The estimates appear to be of good accuracy for all wind state variables, although some small errors affect the two angles. The reason for this behavior can be traced back to mild nonlinearities clearly not captured by the linear model that affect angles to a greater extent than shears. Among the wind parameters, the upflow seems to be the least accurate, while the horizontal shear appears as the most precise. Similar results not shown here were obtained for different wind speeds and flow conditions.

The match improves with the use of the nonlinear model, as reported in Fig. 7. The plots show that all quantities appear to be well captured, with a clear improvement in the quality of the results.

\section{A priori observability analysis}

The observability of the wind parameters is analyzed next. As one can easily imagine, the level of accuracy of the estimates strongly depends on the sensitivity of the moments with respect to the to-be-estimated parameters and the noise in the measurements.

Assuming a linear model, the real (unknown) wind state vector $\boldsymbol{\theta}_{\mathrm{R}}$ is related to the measured load vector $\boldsymbol{m}_{\mathrm{M}}$ as

$\boldsymbol{m}_{\mathrm{M}}=\mathbf{F} \boldsymbol{\theta}_{\mathrm{R}}+\boldsymbol{m}_{0}+\boldsymbol{r}$. 

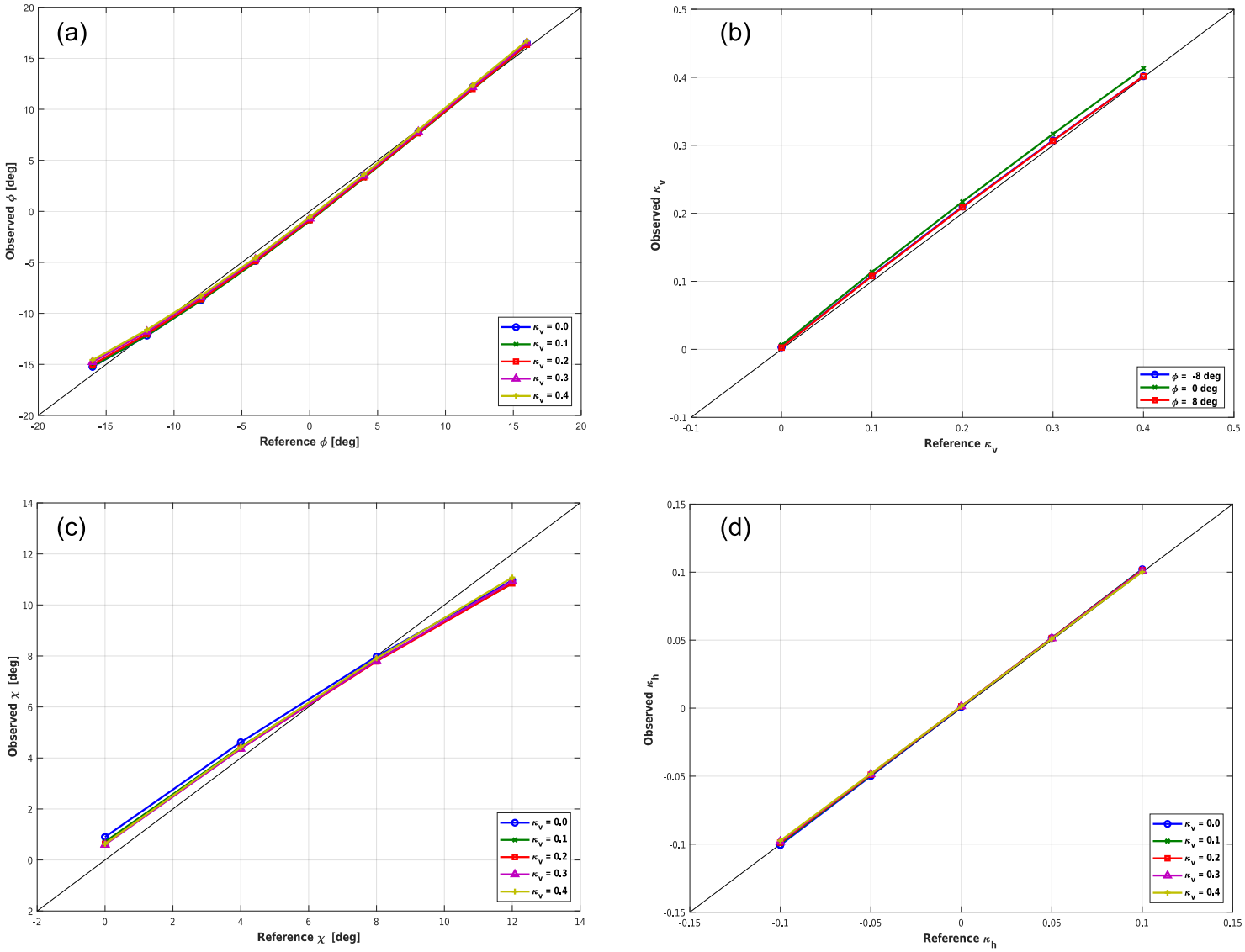

Figure 6. Wind states observed using the linear model for different steady inflow conditions at $5 \mathrm{~m} \mathrm{~s}^{-1}$ : yaw misalignment $\phi$ at $\chi=8^{\circ}$ and $\kappa_{\mathrm{h}}=-0.1(\mathbf{a})$, vertical shear $\kappa_{\mathrm{V}}$ at $\chi=8^{\circ}$ and $\kappa_{\mathrm{h}}=-0.1(\mathbf{b})$, upflow angle $\chi$ at $\phi=-8^{\circ}$ and $\kappa_{\mathrm{h}}=-0.1(\mathbf{c})$, horizontal shear $\kappa_{\mathrm{h}}$ at $\chi=8^{\circ}$ and $\phi=-8^{\circ}(\mathbf{d})$.

By inserting Eq. (29) into Eq. (28), the estimation error $\boldsymbol{\epsilon}_{\theta}$ is readily derived as

$\boldsymbol{\epsilon}_{\boldsymbol{\theta}}=\boldsymbol{\theta}_{\mathrm{E}}-\boldsymbol{\theta}_{\mathrm{R}}=\left(\mathbf{F}^{T} \mathbf{R}^{-1} \mathbf{F}\right)^{-1} \mathbf{F}^{T} \mathbf{R}^{-1} \boldsymbol{r}$.

The estimate is unbiased because the expected value of the error $\mathbf{E}\left[\boldsymbol{\epsilon}_{\boldsymbol{\theta}}\right]$ is equal to zero when the residual is zeromean. Additionally, the covariance of the estimation error $\operatorname{Cov}\left[\boldsymbol{\epsilon}_{\boldsymbol{\theta}}\right]=\mathcal{E}\left[\boldsymbol{\epsilon}_{\boldsymbol{\theta}} \boldsymbol{\epsilon}_{\boldsymbol{\theta}}{ }^{T}\right]$ (Crámer, 1946) is written as

$\operatorname{Cov}\left[\boldsymbol{\epsilon}_{\boldsymbol{\theta}}\right]=\left(\mathbf{F}^{T} \mathbf{R}^{-1} \mathbf{F}\right)^{-1}$.

This expression shows the interplay between noise $\boldsymbol{r}$ and sensitivity $\mathbf{F}$ captured by the term $\mathbf{R}^{-\frac{1}{2}} \mathbf{F}$ : the higher the variance and/or the lower the sensitivity of the measurements with respect to the wind states, the worse the accuracy of the estimates.

The covariance $\operatorname{Cov}\left[\boldsymbol{\epsilon}_{\boldsymbol{\theta}}\right]$ expressed by Eq. (31) is typically fully populated, as the errors of the estimates are correlated. To ease the understanding of the estimation problem, the SVD (Golub and van Loan, 1996) can be used to decouple the estimates. In fact, matrix $\mathbf{R}^{-\frac{1}{2}} \mathbf{F}$ can be factored as

$\mathbf{R}^{-\frac{1}{2}} \mathbf{F}=\mathbf{U} \mathbf{\Sigma} \mathbf{V}^{T}$,

with $\mathbf{U} \in \mathfrak{R}^{m \times m}, \quad \boldsymbol{\Sigma} \in \mathfrak{R}^{m \times n}$ and $\mathbf{V} \in \mathfrak{R}^{n \times n}$, where $m$ is the number of measurements and $n$ is the number of wind state variables. Matrices $\mathbf{U}$ and $\mathbf{V}$ are orthonormal, i.e., $\mathbf{U}^{T} \mathbf{U}=\mathbf{U U}^{T}=\mathbf{I}$ and $\mathbf{V}^{T} \mathbf{V}=\mathbf{V} \mathbf{V}^{T}=\mathbf{I}$, whereas $\boldsymbol{\Sigma}=$ $\operatorname{diag}\left(\ldots, 1 / \sigma_{i}, \ldots\right)$ is a diagonal matrix and $\sigma_{i}$ is the standard deviation. By inserting Eq. (32) into Eq. (31), the covariance of the estimation error can be expressed as

$$
\begin{aligned}
\operatorname{Cov}\left[\mathbf{V}^{T} \boldsymbol{\epsilon}_{\theta}\right] & =\boldsymbol{E}\left[\left(\mathbf{V}^{T}\left(\boldsymbol{\theta}_{\mathrm{E}}-\boldsymbol{\theta}_{\mathbf{R}}\right)\right)\left(\mathbf{V}^{T}\left(\boldsymbol{\theta}_{\mathrm{E}}-\boldsymbol{\theta}_{\mathbf{R}}\right)\right)^{T}\right] \\
& =\left(\boldsymbol{\Sigma}^{T} \boldsymbol{\Sigma}\right)^{-1}=\operatorname{diag}\left(\ldots, \sigma_{i}{ }^{2}, \ldots\right) .
\end{aligned}
$$

This way, the problem is reformulated by the change in variables $\boldsymbol{\xi}=\mathbf{V}^{T} \boldsymbol{\theta}$, where $\boldsymbol{\xi}$ variables are statistically independent with diagonal covariance. This reformulation simplifies the interpretation of the structural observability of the problem. In fact, the $i$ th column of matrix $\mathbf{V}$ linearly combines the wind parameters by mapping them into a new parameter 

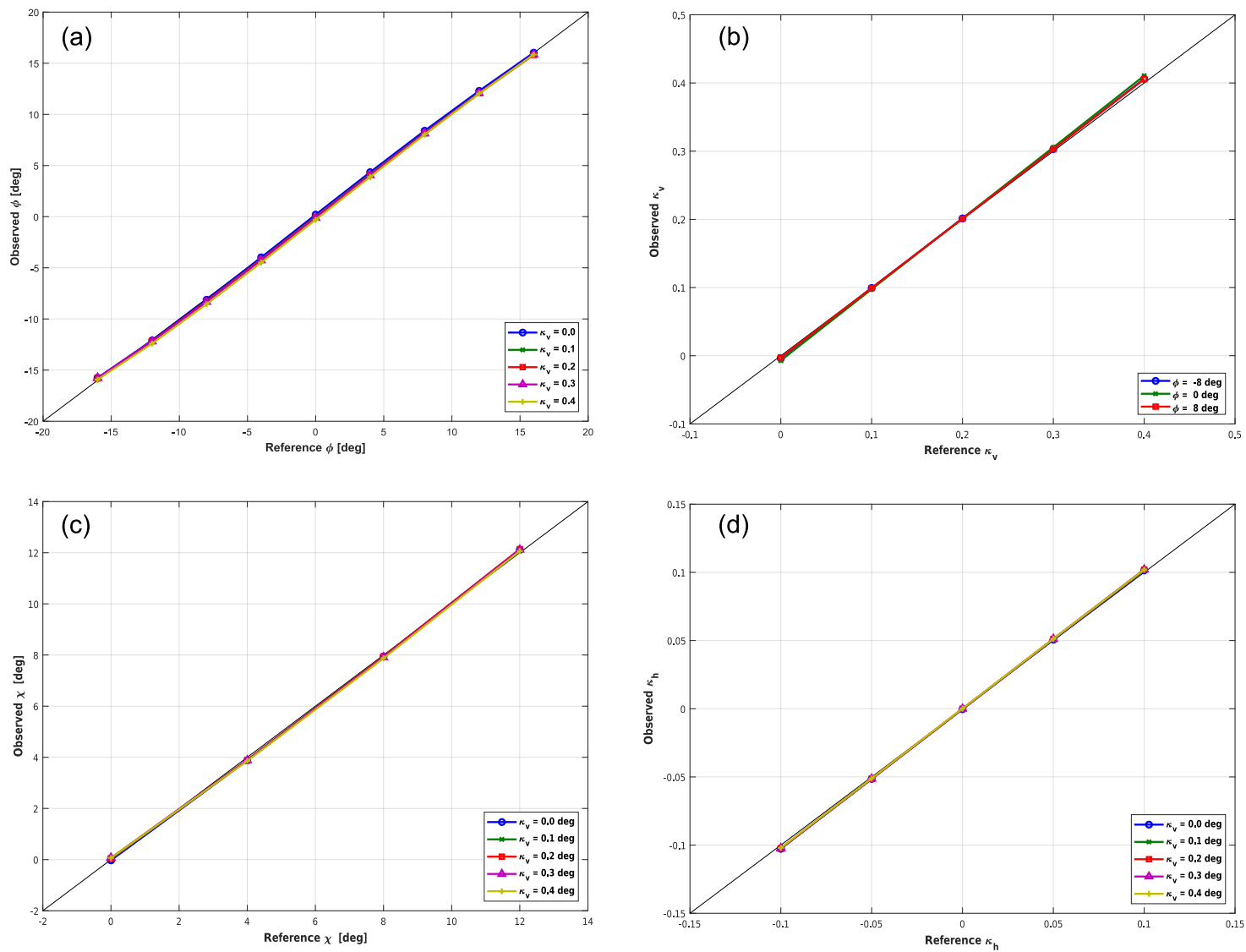

Figure 7. Wind state observed with the nonlinear model for different steady inflow conditions at $5 \mathrm{~m} \mathrm{~s}^{-1}$ : yaw misalignment $\phi$ at $\chi=8^{\circ}$ and $\kappa_{\mathrm{h}}=-0.1(\mathbf{a})$, vertical shear $\kappa_{\mathrm{V}}$ at $\chi=8^{\circ}$ and $\kappa_{\mathrm{h}}=-0.1(\mathbf{b})$, upflow angle $\chi$ at $\phi=-8^{\circ}$ and $\kappa_{\mathrm{h}}=-0.1$ (c), horizontal shear $\kappa_{\mathrm{h}}$ at $\chi=8^{\circ}$ and $\phi=-8^{\circ}(\mathbf{d})$.

$\xi_{i}$ with variance $\sigma_{i}{ }^{2}$. Clearly, a high variance indicates a low level of identifiability of the associated linear combination of wind parameters.

This analysis also provides information on the dependence of loads on wind states. In fact, one can easily show that

$\frac{\partial \boldsymbol{m}}{\partial \boldsymbol{\xi}}=\mathbf{R}^{\frac{1}{2}} \mathbf{U} \boldsymbol{\Sigma}$.

Therefore, the analysis of $\mathbf{U}$ reveals the linear combination of inflow parameters on which each load depends the most.

The same analysis can be applied to the nonlinear case by linearizing Eq. (21) around a specific operating and wind condition and using $\mathbf{F}=\partial\left(\boldsymbol{F}_{\mathbf{N L}} \theta_{\mathbf{N L}}\right) / \partial \boldsymbol{\theta}=\boldsymbol{F}_{\mathbf{N L}} \partial \theta_{\mathbf{N L}} / \partial \boldsymbol{\theta}$.

\subsection{Results of the a priori analysis}

The a priori analysis was applied to the identified inputoutput model. Three different values of the noise covariance $\mathbf{R}$ were considered. In the first two cases, all measures were assumed to be uncorrelated and affected by the same noise level, i.e., $\mathbf{R}=\gamma^{2} \mathbf{I}$, where $\gamma$ is a positive real number. In the first case, $\gamma$ was set to $0.01 m_{\min }$, with $m_{\min }$ being the minimum of the load amplitude maxima. In the second case, $\gamma$ was set to $0.01 m_{\max }$, with $m_{\max }$ being the maximum of the load amplitude maxima. In the third case, the noise covariance was computed using Eq. (26):

$$
\begin{aligned}
\mathbf{R}_{\epsilon} & =\frac{1}{N_{\exp }} \sum_{i=1}^{N_{\exp }} \boldsymbol{r}_{i} \boldsymbol{r}_{i} T \\
& =\frac{1}{N_{\exp }} \sum_{i=1}^{N_{\exp }}\left(\boldsymbol{m}_{\mathrm{M} i}-\mathcal{M}_{i}(\boldsymbol{\theta}, V, \varrho)\right) \\
& \left(\boldsymbol{m}_{\mathrm{M} i}-\mathcal{M}_{i}(\boldsymbol{\theta}, V, \varrho)\right)^{T}
\end{aligned}
$$

where $\boldsymbol{m}_{\mathrm{M} i}$ values are loads measured on the simulation model and $\mathcal{M}_{i}(\boldsymbol{\theta}, V, \varrho)$ represents those given by the observation model.

For the first case, matrices $\mathbf{V}$ and $\mathbf{U}$ were computed at a wind speed of $5 \mathrm{~m} \mathrm{~s}^{-1}$ to obtain

$$
\mathbf{V}=\left[\begin{array}{cccc}
\sim 0 & \sim 0 & \mathbf{0 . 5 5} & \mathbf{0 . 8 3} \\
\sim 0 & \sim \mathbf{1} & \sim 0 & \sim 0 \\
\sim 0 & \sim 0 & \mathbf{0 . 8 3} & \mathbf{0 . 5 5} \\
\sim \mathbf{1} & \sim 0 & \sim 0 & \sim 0
\end{array}\right],
$$



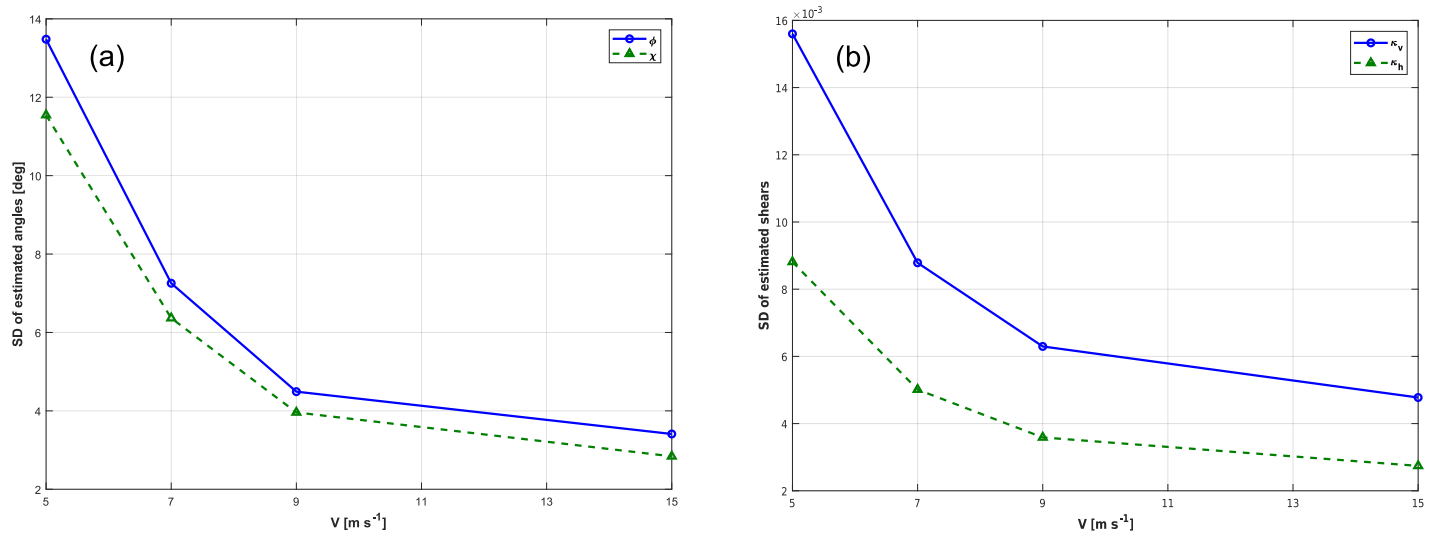

Figure 8. Expected standard deviation of the wind state estimates as a function of wind speed. (a) Standard deviations for angles $\phi$ and $\chi$; (b) standard deviations for shears $\kappa_{\mathrm{V}}$ and $\kappa_{\mathrm{h}}$.

$\mathbf{U}=\left[\begin{array}{cccc}0.11 & 0.97 & 0.14 & 0.18 \\ -0.97 & 0.11 & 0.18 & -0.14 \\ 0.03 & 0.22 & -0.60 & -0.77 \\ -0.23 & 0.02 & -0.77 & 0.60\end{array}\right]$

where $\sim 0$ and $\sim 1$ indicate a number approximately equal to 0 and to 1 , respectively. To interpret these results, remember that the wind state vector is defined as $\boldsymbol{\theta}=$ $\left(\phi, \kappa_{\mathrm{v}}, \chi, \kappa_{\mathrm{h}}\right)^{T}$, whereas the load vector is defined as $\boldsymbol{m}=$ $\left(m_{1 \mathrm{c}} \mathrm{OP}, m_{1 \mathrm{~s}} \mathrm{OP} m_{1 \mathrm{c}} \mathrm{IP}, m_{1 \mathrm{~s}} \mathrm{IP}^{T}\right.$.

The first and second columns of $\mathbf{V}$ are related to the horizontal and vertical shears, respectively. Since their maximum entries approach 1, both parameters can be independently identified. However, a coupling between the two angles can be seen in the third and fourth columns: an error in the estimation of one angle will propagate and affect the estimate of the other. Similar $\mathbf{V}$ matrices leading to the same conclusions were computed at different wind speeds and different noise levels $\gamma^{2}$.

To interpret matrix $\mathbf{U}$, consider that rows are associated with entries of the load vector, whereas columns are associated with entries of the wind state vector. The first column of $\mathbf{U}$ shows that the horizontal shear mostly affects the sine components of both out- and in-plane moments. Similarly, the second column shows that the vertical shear mostly affects the cosine components of the loads. In contrast, the third and fourth columns, associated with the angles, do not indicate a predominant effect on some load components. In fact, all loads are affected by both upflow and yaw misalignment, with the in-plane harmonics exhibiting higher sensitivity.

As a side observation, also note the symmetry between the couples $\left\{\phi, \kappa_{\mathrm{v}}\right\}$ and $\left\{\chi, \kappa_{\mathrm{h}}\right\}$, which is an effect of the nearly $90^{\circ}$ symmetry in the definition of the wind parameters and in the response of the machine (see Fig. 1). In other words, for the same horizontal or vertical shear the rotor response will be the same but shifted by $90^{\circ}$. Similarly, for the same upflow
Table 1. Expected variance of wind state estimates based on the a priori analysis.

\begin{tabular}{lrrr}
\hline Standard deviations & $0.01 m_{\min }$ & $0.01 m_{\max }$ & $\mathbf{R}_{\epsilon}$ \\
\hline$\sigma_{\phi}[\mathrm{deg}]$ & 0.95 & 26.0 & 2.5 \\
$\sigma_{\kappa_{\mathrm{v}}}$ & $1.1 \times 10^{-3}$ & $3.0 \times 10^{-2}$ & $6.5 \times 10^{-2}$ \\
$\sigma_{\chi}[\mathrm{deg}]$ & 0.81 & 22.3 & 1.5 \\
$\sigma_{\kappa_{\mathrm{h}}}$ & $6.2 \times 10^{-4}$ & $1.7 \times 10^{-2}$ & $2.3 \times 10^{-2}$ \\
\hline
\end{tabular}

and misalignment angles, the rotor response will be the same with a $90^{\circ}$ shift. This symmetry in the behavior of the rotor can be exploited to simplify the identification problem, as shown in Cacciola et al. (2016a).

Table 1 reports the expected variances of the wind state estimation errors for the three considered noise variances. It appears that, as expected, higher noise levels are associated with higher variances of the estimates. In addition, the variance of the angles appears to be significantly higher than that of the shears. In fact, angle variances approach and exceed tens of degrees for the higher noise levels, indicating that instantaneous estimates of these wind states are probably impractical. However, longer-term observation could be possible with time filtering, as discussed and shown later.

Finally, Fig. 8 shows the standard deviation (SD) of the wind parameter estimates with respect to the wind speed, computed assuming $\mathbf{R}=\gamma \mathbf{I}$ with $\gamma=0.01\left(m_{\min }+m_{\max }\right) / 2$. The plot shows a marked improvement in the quality of the estimates with wind speed.

Similar results not shown here for the sake of brevity were obtained with the nonlinear model.

\subsection{Expected observer behavior}

Given the behavior of the linear and nonlinear observers and the results of the SVD-based a priori observability analysis, the following conclusions can be made. 


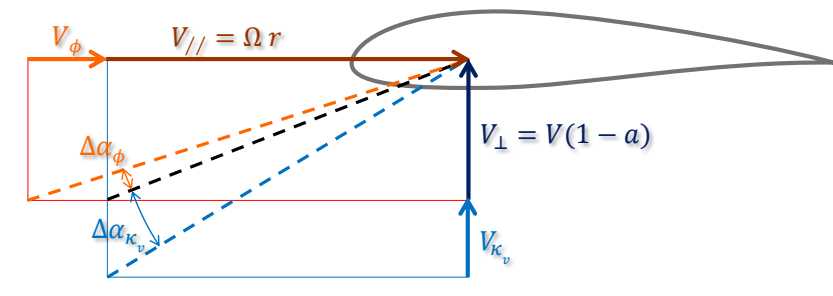

Figure 9. Effects of shear and misalignment changes on sectional angle of attack.

- In general, it should be possible to estimate both shears with satisfactory precision, as their errors are moderate even for significant measurement noise levels.

- It is expected that the estimation of both yaw misalignment and upflow angle will be more significantly affected by measurement noise. Because of this, the estimation of these angles should be accompanied by a suitable filtering action in order to remove fast fluctuations. This also implies that these angles can only be estimated on longer time horizons than in the case of shears.

- The observation accuracy should increase with increasing wind speed.

- The nonlinear model appears to be more accurate than the linear model for the estimation of yaw misalignment and upflow angles. However, shears also seem to be captured well by the linear model.

The different expected accuracy in the estimation of shears and angles can be given an even more intuitive explanation. Consider the blade section depicted in Fig. 9. The relative airflow velocity vector can be decomposed into the component $V_{\perp}=(1-a) V$ perpendicular to the rotor disk plane, where $a$ is the local induction factor and the one $V_{/ /}=\Omega r$ parallel to it, with $r$ being the section radial position.

A change in shear will be seen by the blade section mainly as a change in $V_{\perp}$. However, a change in misalignment will induce a change mainly in $V_{/ /}$. The figure shows that two equal velocity perturbations, $\Delta V=V_{\kappa_{\mathrm{v}}}=V \sin (\phi)$ perpendicular and parallel to the rotor plane, respectively, will induce different changes in the sectional angle of attack. In particular, the change due to perpendicular (shear-caused) variation is larger than that due to parallel (misalignment-caused) variation.

This is also easily shown by considering that the inflow angle is $\tan \zeta=V_{\perp} / V_{/ /}$. Hence, for a perturbation $\Delta V$ due to shear variation, the inflow changes as $\tan \zeta=\left(V_{\perp}+\right.$ $\Delta V) / V_{/ /}$. In contrast, for a perturbation $\Delta V$ due to misalignment variation, the inflow changes as $\tan \zeta=V_{\perp} /\left(V_{/ /}+\right.$ $\Delta V)$. For typical values of $V_{\perp}$ and $V_{/ /}$, Fig. 10 shows the behavior of $\tan \zeta$ as a function of $\Delta V$. As clearly shown by the plot, for a same perturbation $\Delta V$ (for example, $1 \mathrm{~m} \mathrm{~s}^{-1}$,

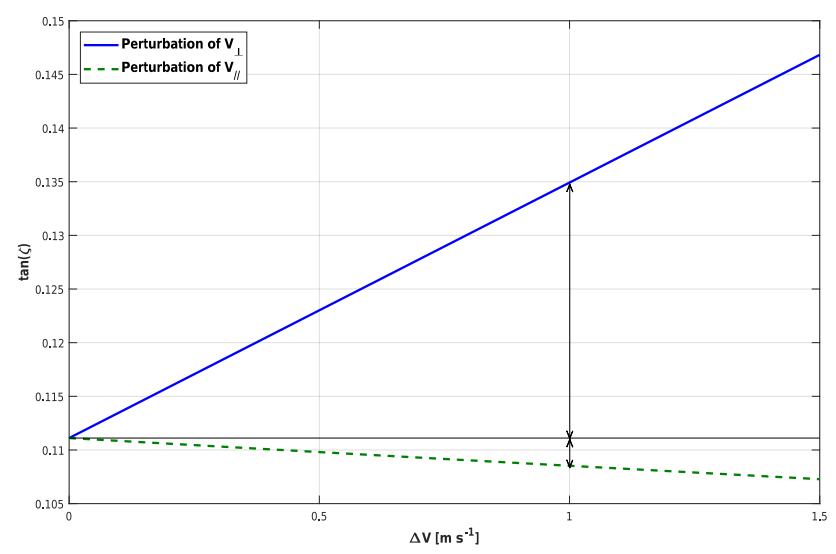

Figure 10. Variation in the inflow angle $\zeta$ at a blade section as a function of a perturbation $\Delta V$ in a direction either perpendicular (solid line) or parallel (dashed line) to the rotor plane.

as shown in the figure), the ensuing change in inflow angle is larger when the perturbation is due to a change in shear than when it is due to a change in misalignment. This implies similarly larger variation in the sectional angle of attack and hence in the loads. In conclusion, one may expect that the rotor response will be more sensitive to variations in shear than in misalignment when these two different phenomena produce velocity perturbations of the same magnitude. Due to the rotational symmetry of the problem, the same conclusions clearly hold true for variation in horizontal shear or for variation in the vertical upflow angle.

\section{Results}

After having verified in the previous sections that blade load harmonics carry enough information to infer wind states in steady conditions, attention is now turned to the dynamic problem. The nonturbulent case is considered first by using fully parameterized wind fields with variable-in-time wind states. Next, the turbulent case is considered by using wind fields modeled by the Kaimal method for different constant mean wind states. Finally, turbulent conditions with variablein-time mean quantities are considered.

\subsection{Nonturbulent case with fully parameterized wind fields}

Ideal nonturbulent and fully parameterized wind fields with time-varying wind states were generated according to Eq. (3) by independently varying angles $\phi$ and $\chi$ as well as shears $\kappa_{\mathrm{v}}$ and $\kappa_{\mathrm{h}}$. Here and in the following examples, load harmonics were extracted from the simulated wind turbine response by using the Coleman transformation followed by filtering with an eighth-order Butterworth filter with a cut-out frequency equal to $0.35 f_{\text {tower }}=0.105 \mathrm{~Hz}$ to remove load oscillations at the tower frequency. Finally, inflow conditions were es- 
timated with the proposed observer and compared with the real ones.

Figure $11 \mathrm{a}$ and $\mathrm{b}$ show the results obtained at 4 and $9 \mathrm{~m} \mathrm{~s}^{-1}$, respectively, using the linear and nonlinear models. The agreement is generally good as all parameters are well observed by both models. The observed states are affected by a delay of about $7 \mathrm{~s}$, primarily due to the effects of the filter. There are minor differences between the linear and the nonlinear models, which are not large enough to allow any conclusions to be drawn.

\subsection{Turbulent case}

Different turbulent wind fields were generated using the TurbS im software according to the Kaimal model. The corresponding inflow conditions, in terms of hub-height wind speed $V$ and wind states $\boldsymbol{\theta}$, were then computed by fitting at each instant in time the wind state parameterization (Eq. 1) to the turbulent wind grid over the complete rotor disk. The wind parameters obtained this way were then used as reference quantities to verify the accuracy of the estimated ones.

As wind states are inferred from blade loads, which in turn depend on the wind conditions at the location occupied by each single blade at each time instant, an alternative way of computing the reference wind conditions was also used. In this second implementation, wind parameters were computed by fitting the wind state parameterization expressed by Eqs. (2) and (3) not over the complete rotor disk, but only to its portion occupied at that time instant by the three blades. Spanwise weighting was also used on account of the nonuniform power extraction characteristics of rotors (Soltani et al., 2013). As the two methods do not yield significantly different reference wind states, only the results obtained with the first approach are reported in the following.

Figures 12 and 13 report the results obtained at 7 and $19 \mathrm{~m} \mathrm{~s}^{-1}$, which respectively belong to regions II and III. Figures $12 \mathrm{a}$ and 13a show the results for a TI equal to $2 \%$, and Figs. $12 \mathrm{~b}$ and $13 \mathrm{~b}$ show the $12 \%$ TI case.

These results suggest several possible considerations.

First, the estimates of both shears $\kappa_{\mathrm{v}}$ and $\kappa_{\mathrm{h}}$ appear to have generally high accuracy: their mean values and their rapid oscillations are well captured by both the linear and nonlinear models. Here again, the results are affected by a $7 \mathrm{~s}$ delay induced by the filter. For the lower wind speeds and turbulence intensities, the linear and nonlinear observers exhibit very similar behavior. However, differences appear at $19 \mathrm{~m} \mathrm{~s}^{-1}$ and $12 \% \mathrm{TI}$, as shown by Fig. 13b. In fact, between second 250 and second 350 of the simulation, the estimation of the vertical shear provided by the linear model is affected by large errors, whereas the nonlinear observer results still remain acceptable.

The good behavior of the shear estimates suggests the possible use of a faster filter in order to reduce the estimation delay. For example, the delay can be reduced to only $4 \mathrm{~s}$ by us- ing a filter cut-out frequency of $0.17 \mathrm{~Hz}$, which corresponds to 1.2 times the rotor frequency at $5 \mathrm{~m} \mathrm{~s}^{-1}$.

An estimation of the angles $\phi$ and $\chi$ does not prove to be as accurate as the estimation of the shears, as fully expected based on the a priori observability study. Mean values are well captured, especially by the nonlinear model, but fluctuations are missed by both observers.

The general lower quality of the estimates for the angles was previously explained by the a priori analysis, and it is clearly illustrated a posteriori by the simulation results shown here. Various sources of error may ultimately be responsible for the oscillations in the estimates shown by the plots, including unmodeled dynamics, rapid pitch motions or variable rotor speed. It is interesting to recall that the steady model (Eq. 9) appeared capable of capturing the behavior of the $1 \times$ Rev loads in turbulent conditions, as clearly illustrated by the results shown in Fig. 5a. Notwithstanding this apparently more than satisfactory behavior when used to simulate loads given wind states, the inversion of the model to yield wind states given loads appears to be more problematic. In fact, because of the generally lower level of observability of the angles with respect to the shears (see Sect. 3), errors propagate throughout the solution at a high rate for wind misalignment and upflow, in turn generating fast fluctuations of the estimates.

It should also be remarked that an additional source of uncertainty is the ground truth. In fact, the presence of turbulent eddies in the flow implies that the wind field cannot be exactly parameterized by the assumed wind states. Hence, the reference quantities plotted here should also be considered only as indicative proxies of the actual wind states.

The observation errors were further analyzed from a statistical standpoint by generating five different 10 min turbulent wind field realizations and computing means and standard deviations. To eliminate the effects of the delay caused by the filter, which would have prevented any instantaneous comparison between the reference and observed quantities, reference wind states were processed with the same filter used for the moment harmonics.

Figure 14 shows the behavior of the standard deviation of the estimation error for the four wind states as functions of the wind speed and for different TI levels. The curves labeled "TI $=0 \%$ " refer to the nonturbulent fully parameterized conditions already described in Sect. 4.1. Since similar behavior characterizes the results of both observers, only those obtained with the nonlinear model are shown here to avoid cluttering the figure.

As expected, the standard deviation increases with the TI level. Moreover, in regions II and II $\frac{1}{2}$, accuracy tends to increase for increasing wind speed, as similarly predicted by the a priori observability analysis. The opposite happens in region III, where oscillations in the results are more significant and strongly affect the estimates. This behavior is particularly visible in the estimation of the angles, as shown in Fig. 14a and c. 

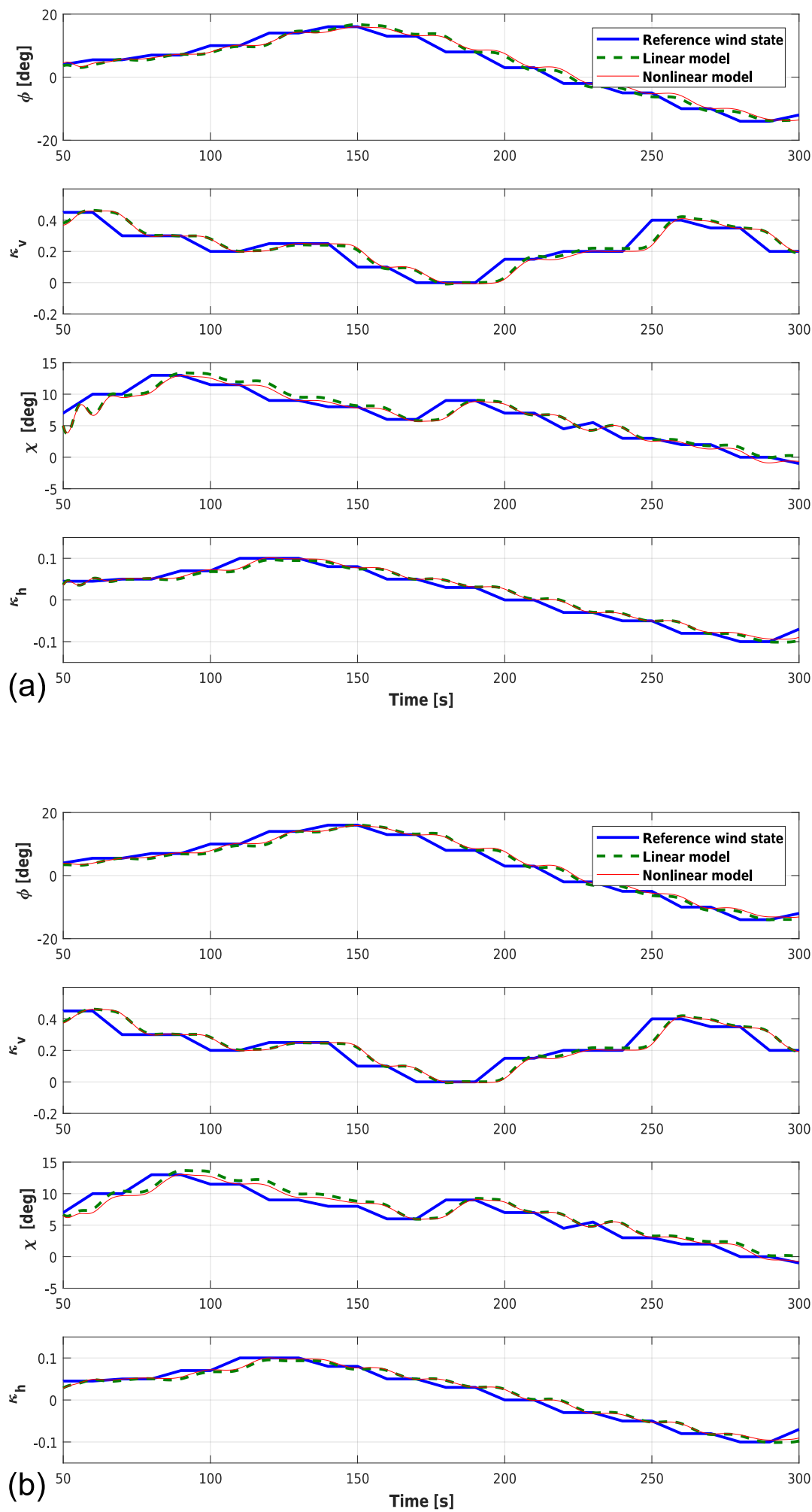

Figure 11. Wind state observations in nonturbulent wind conditions with variable wind parameters at 4 (a) and $9 \mathrm{~m} \mathrm{~s}^{-1}$ (b). Solid thick blue lines: real wind parameters; dashed thick green lines: observations by the linear model; solid thin red lines: observations by the nonlinear model. 

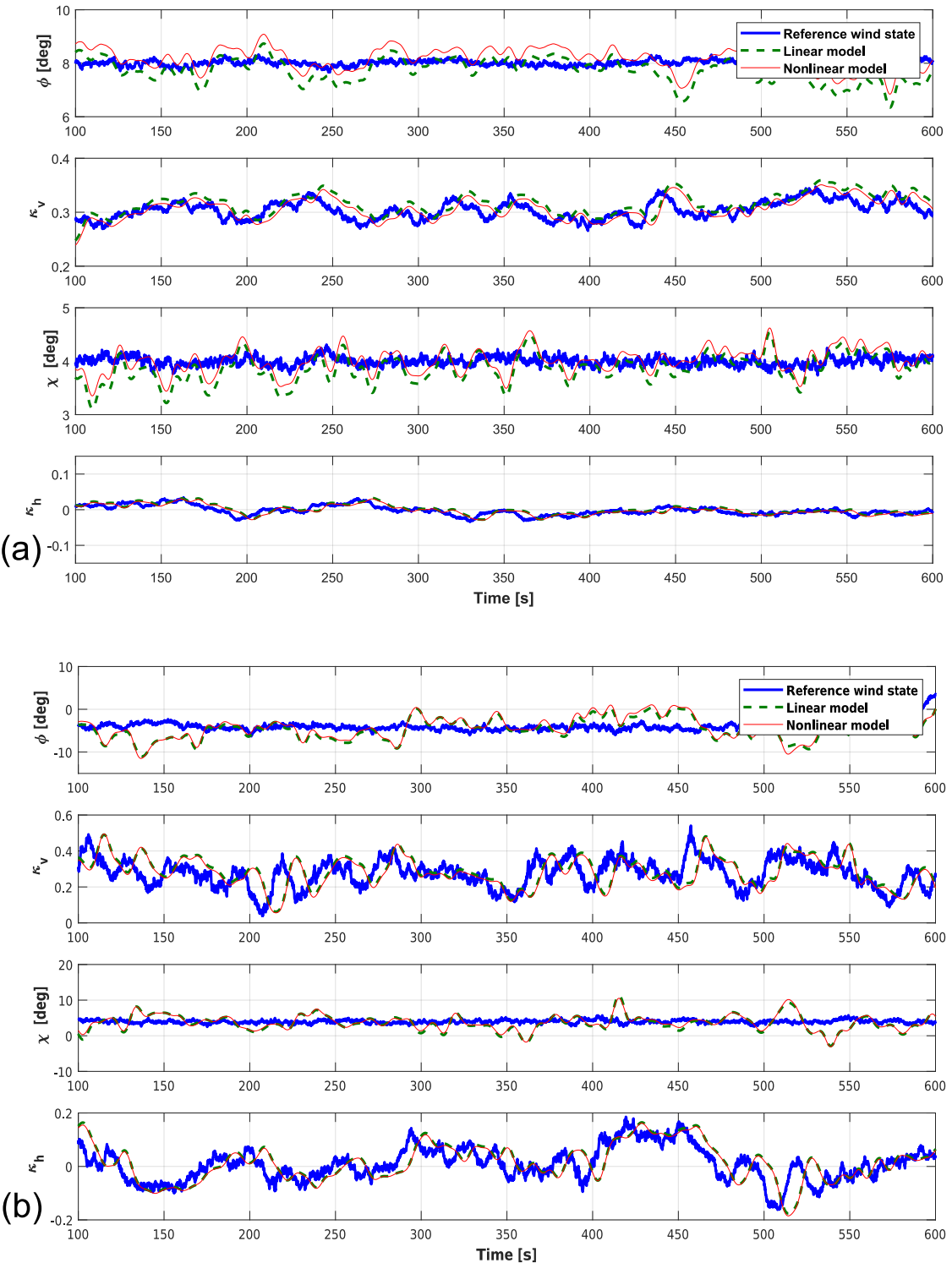

Figure 12. Wind state observations in turbulent wind conditions at $7 \mathrm{~m} \mathrm{~s}^{-1}$ for a TI equal to 2 (a) and $12 \%$ (b). Solid thick blue lines: reference wind parameters; dashed thick green lines: observations by the linear model; solid thin red lines: observations by the nonlinear model.

Shear errors also remain low at very high TI levels, as illustrated by Fig. 14b and d, indicating that fast, good-quality shear estimates are possible. In fact, for example, the standard deviation of $\kappa_{\mathrm{V}}$ at $7 \mathrm{~m} \mathrm{~s}^{-1}$ and $20 \% \mathrm{TI}$ is circa 0.055 , which means that about $95 \%$ of the observer samples have an instantaneous error lower than 0.11 .

The evaluation of the observer performance for the angles deserves special attention. Looking at the yaw misalignment in Fig. 14a for regions II, II $\frac{1}{2}$ and the low region III up to $15 \mathrm{~m} \mathrm{~s}^{-1}$, the instantaneous error remains within acceptable bounds for turbulence intensities lower than $5 \%$. In fact, $\sigma_{\phi}$ is lower than $1.5^{\circ}$, which implies that estimates are affected by an error lower than $3^{\circ} 95 \%$ of the time. On the contrary, the estimation error standard deviation may reach 3,4 or even $6^{\circ}$ for the higher turbulence intensities of 12,16 and $20 \%$. The maximum error deviation is obtained at $19 \mathrm{~m} \mathrm{~s}^{-1}$ for a TI of $12 \%$. The same considerations can be derived for the estimation of the upflow angle.

Figure 15 reports the mean observation errors with respect to the wind speed for both the linear and nonlinear observers. Not unexpectedly, the estimation of the shears is characterized by almost negligible error means. More surprisingly, however, even the mean errors of the angles are quite low for all conditions, although a mild reduction of accuracy can be observed for increasing wind speeds. In addition, as previously noted, the nonlinear observer appears to be slightly 

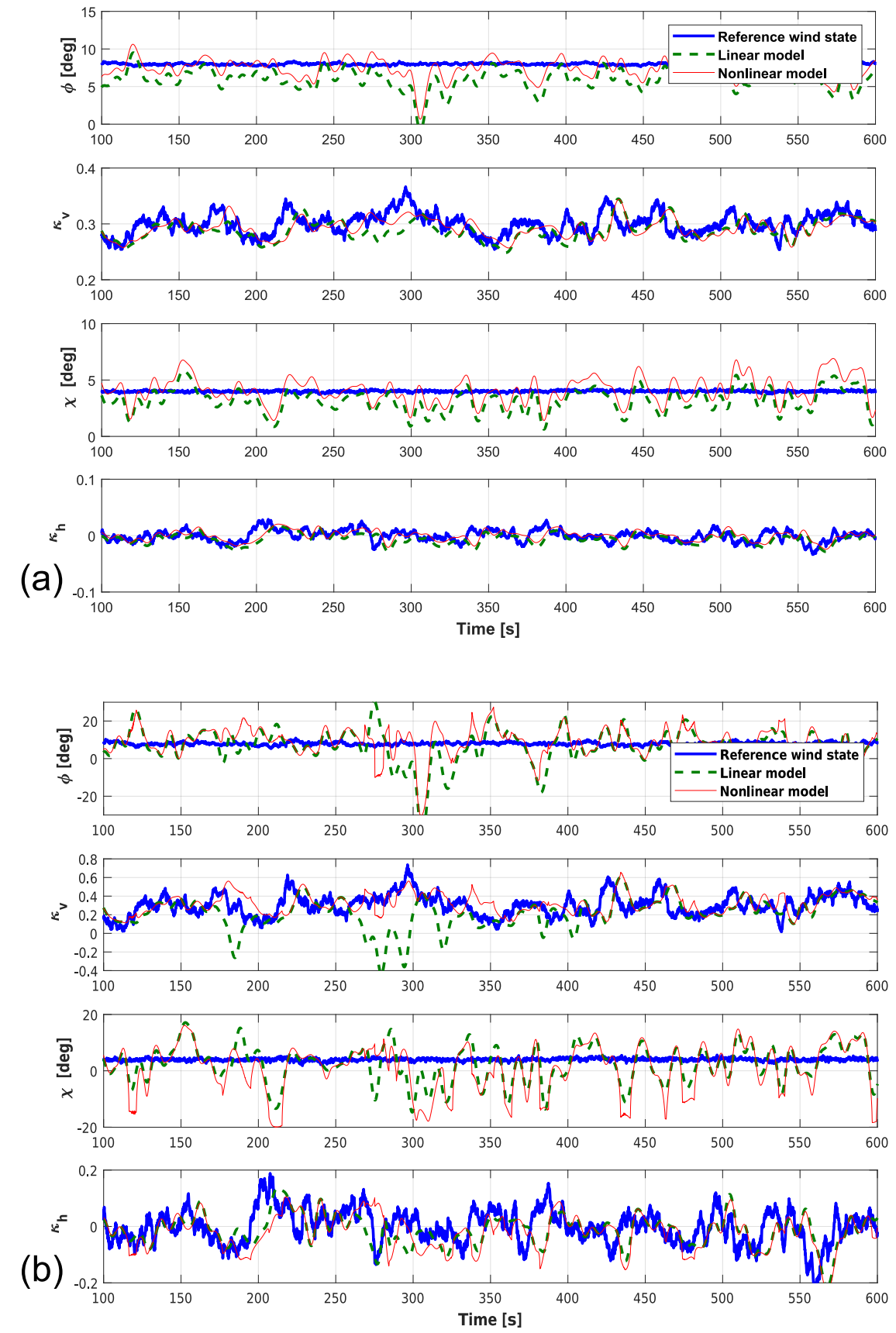

Figure 13. Wind state observations in turbulent wind conditions at $19 \mathrm{~m} \mathrm{~s}^{-1}$ for a TI equal to 2 (a) and $12 \%$ (b). Solid thick blue lines: reference wind parameters; dashed thick green lines: observations by the linear model; solid thin red lines: observations by the nonlinear model.

more accurate than the linear one. As a final remark, it was found that the error means are not significantly influenced by TI. Hence, the TI-dependent curves were not displayed in Fig. 15 to avoid cluttering the plot.

\subsubsection{Evaluation of lifetime performance}

The previous examples have shown that observed angles are typically affected by spurious oscillations for the reasons explained by the a priori analysis. The same examples, however, have also shown that mean values are typically well captured and that the amplitude of oscillations is related to TI. This seems to indicate that fast accurate observations 

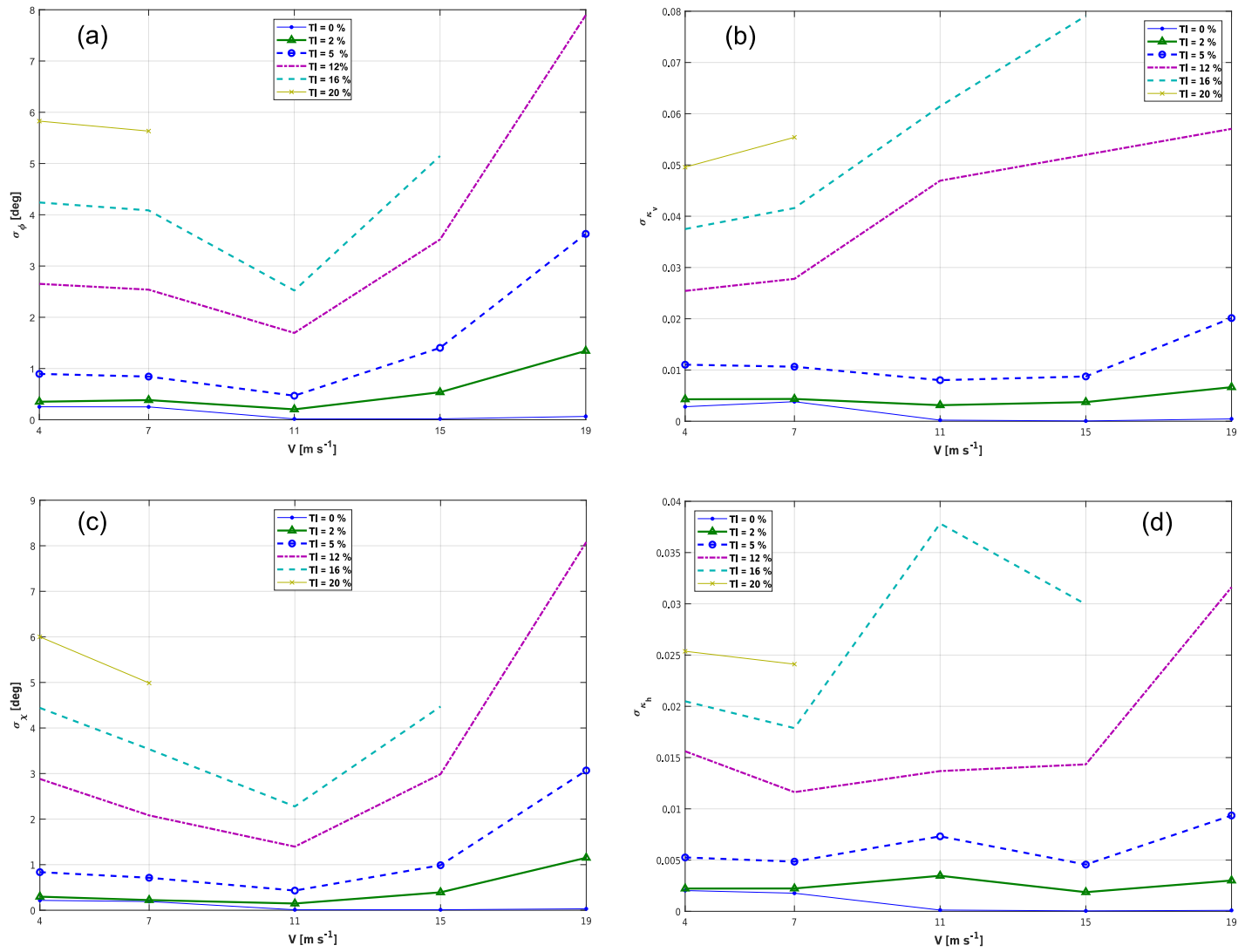

Figure 14. Standard deviation of the estimation error of yaw misalignment (a), vertical shear (b), upflow angle (c) and horizontal shear (d) as functions of wind speed for varying TI levels. All curves refer to the nonlinear observer results.

of angles are generally not possible, while observations on longer time windows might still be relatively accurate. By simple inspection of the temporal responses, it is, however, not easy to get a clear idea of the actual precision of the observers in turbulent conditions. In order to provide a more meaningful indication of the observer accuracy, the "lifetime" standard deviation of the observed states is evaluated in this section. This is computed by weighting the results at each wind speed and TI with the corresponding probability distributions at a given site. To this end, measurements taken at the offshore platform FINO1 (FINO, 2017) from September 2003 to August 2007 were considered. In fact, given the standard deviation of the observation error as a function of $\mathrm{TI}$ and wind speed (given in Fig. 14) and the wind statistics of a specific site, one can evaluate the observer performance when applied to the wind turbine used in the present study if it were located at that site.

Figure 16 shows some statistical metrics of the wind at an altitude over the water line of $80 \mathrm{~m}$, which corresponds to the hub height of the wind turbine considered in the present study. The TI percentiles at $90 \mathrm{~m}$ were extracted from Fig. 2 of Türk and Eimeis (2010) and mapped to the current hub height by scaling with a factor equal to 1.028 according to
Fig. 5.21 of Emeis (2013), which reports the variation in TI as a function of hub height.

Next, a shifted Weibull probability density function (PDF) $\mathcal{W}_{\tau}$ was fitted to the TI for each wind speed. The PDF is defined as

$\mathcal{W}_{\tau}(\tau, V)=$
$\begin{cases}\frac{\alpha(V)}{\beta(V)}\left(\frac{\left(\tau-\tau_{\min }(V)\right)}{\beta(V)}\right)^{\alpha(V)-1} \mathrm{e}^{-\left(\frac{\left(\tau-\tau_{\min }(V)\right)}{\beta(V)}\right)^{\alpha(V)},}, & \tau \geq \tau_{\min }(V), \\ 0, & \tau<\tau_{\min }(V),\end{cases}$

while its associated cumulative distribution function (CDF) is written as

$\mathfrak{W}_{\tau}(\tau, V)= \begin{cases}1-\mathrm{e}^{-\left(\frac{\left(\tau-\tau_{\min }\right)}{\beta(V)}\right)^{\alpha(V)},}, & \tau \geq \tau_{\min }(V), \\ 0, & \tau<\tau_{\min }(V),\end{cases}$

where $\tau$ is the TI level, $\tau_{\min }(V)$ is its minimum value and $\alpha(V)$ and $\beta(V)$ are the shape and scale parameters, respectively, of the probability density function. Figure 17 represents the Weibull PDF and CDF at $9 \mathrm{~m} \mathrm{~s}^{-1}$.

Given the probability density function of the observation error $\mathcal{P}_{\epsilon}$, the TI PDF $\mathcal{W}_{\tau}$ and the wind speed PDF $\mathcal{W}_{V}$, the 

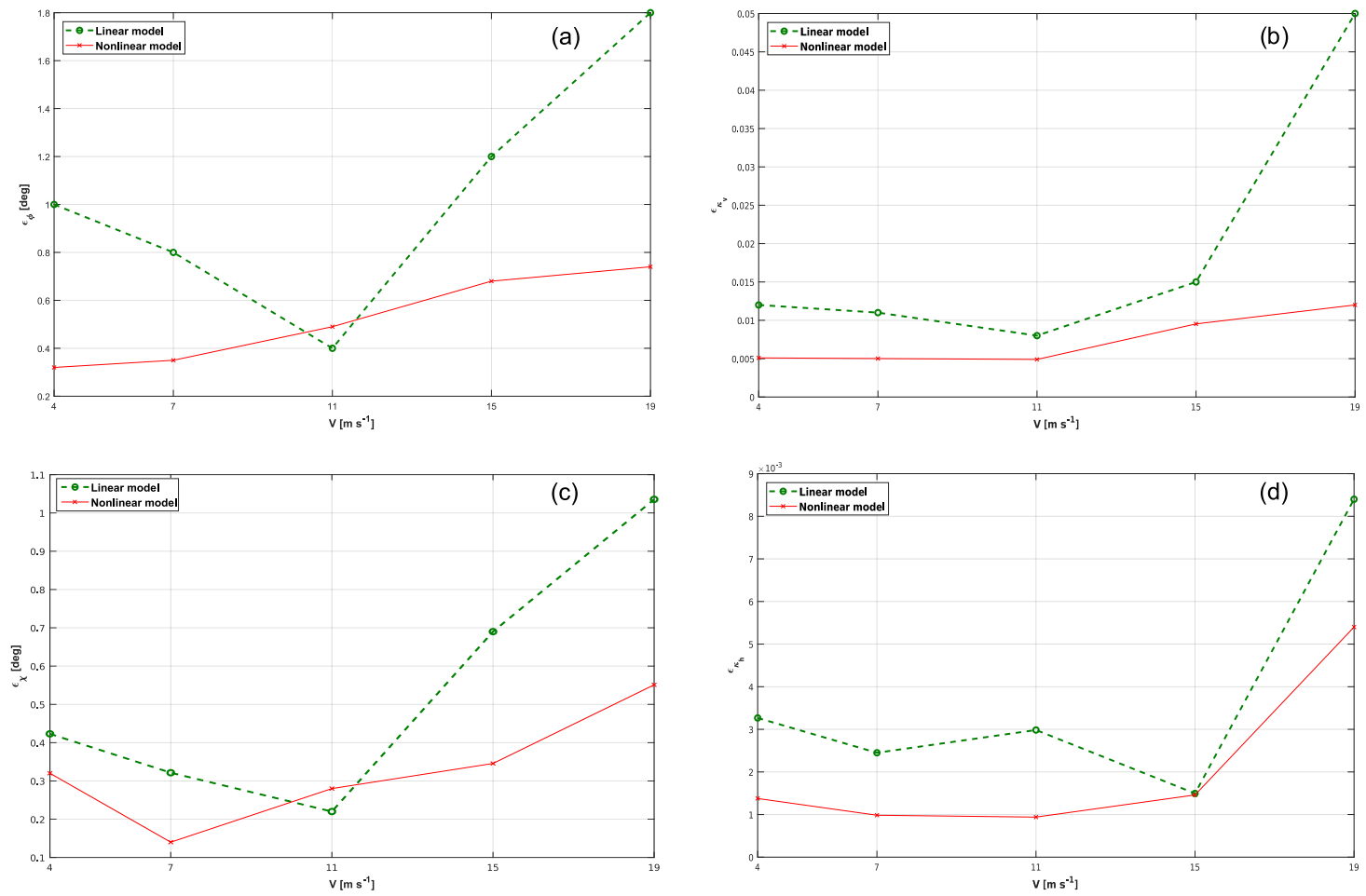

Figure 15. Mean estimation error of yaw misalignment (a), vertical shear (b), upflow angle (c) and horizontal shear (d) with respect to wind speed.

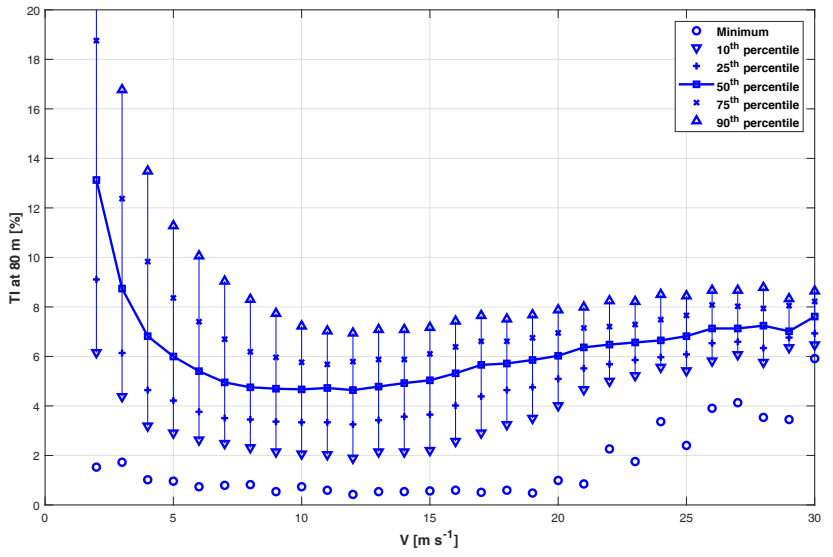

Figure 16. Minimum value, 10th, 25th, 50th, 75th and 90th TI percentiles as functions of wind speed at $80 \mathrm{~m}$ above the water line at FINO1 from September 2003 to August 2007. Data taken from Türk and Eimeis (2010).

lifetime standard deviation $\sigma_{\mathrm{LT}}$ can be readily computed as

$$
\begin{aligned}
\sigma_{\mathrm{LT}}= & \frac{1}{\int_{V_{\mathrm{CI}}}^{V_{\mathrm{CO}}} \mathcal{W}_{V}(V) \mathrm{d} V} \int_{V_{\mathrm{CI}}}^{V_{\mathrm{CO}}} \mathcal{W}_{V}(V) \\
& \left(\int_{0}^{+\infty} \mathcal{W}_{\tau}(V, \tau)\left(\int_{-\infty}^{+\infty} \epsilon \mathcal{P}_{\epsilon}(V, \tau, \epsilon) \mathrm{d} \epsilon\right) \mathrm{d} \tau\right) \mathrm{d} V,
\end{aligned}
$$
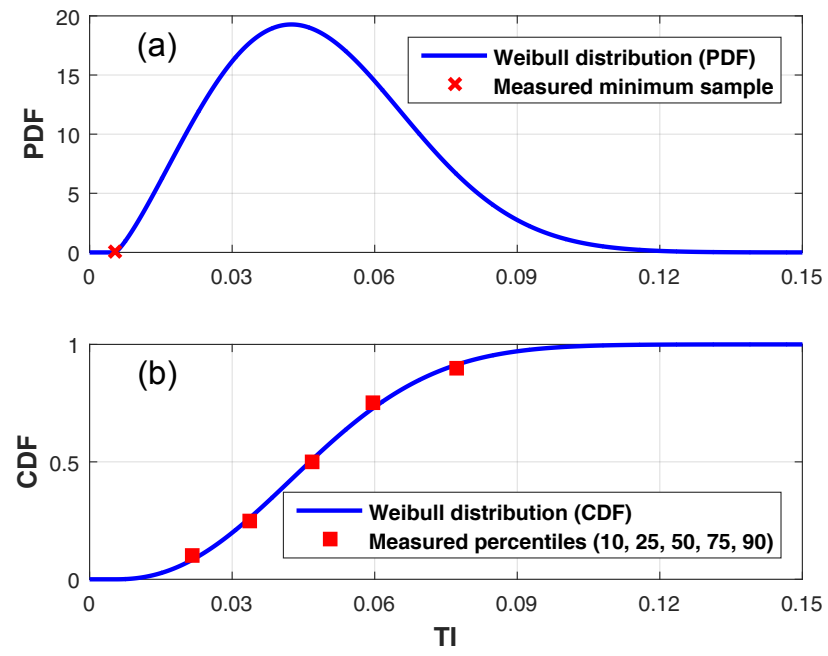

Figure 17. TI PDF (a) and CDF (b) at $9 \mathrm{~m} \mathrm{~s}^{-1}$.

where the innermost integral represents the wind-speedspecific and TI-specific standard deviation of the observation error, $\sigma(V, \tau)$, which was previously computed and reported in Fig. 14. This quantity is then weighted by the probability of each wind speed and TI values to occur at this specific site, as given in Fig. 16. 

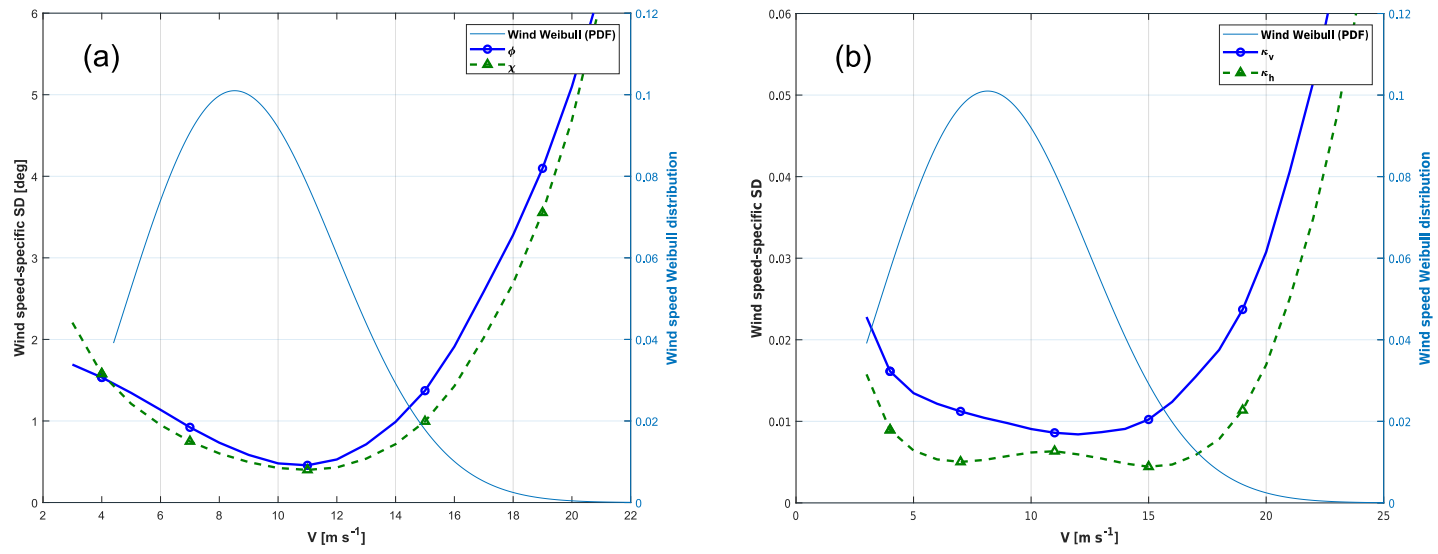

Figure 18. Wind-speed-specific standard deviation of the observation error for angles $\phi$ and $\chi$ (a) and for shears $\kappa_{\mathrm{V}}$ and $\kappa_{\mathrm{h}}(\mathbf{b})$. The wind Weibull distribution is characterized by shape and scale parameters equal to 2.5 and 10 , respectively.

Table 2. Lifetime standard deviation and $2 \sigma$ bounds of the estimation error for the wind parameters.

\begin{tabular}{lrrrr}
\hline Wind parameter & $\begin{array}{r}\phi \\
{[\mathrm{deg}]}\end{array}$ & $\kappa_{\mathrm{V}}$ & $\begin{array}{r}\chi \\
{[\mathrm{deg}]}\end{array}$ & $\kappa_{\mathrm{h}}$ \\
\hline$\sigma_{\mathrm{LT}}$ & 0.97 & 0.011 & 0.84 & 0.006 \\
$2 \sigma_{\mathrm{LT}}$ & 1.94 & 0.022 & 1.68 & 0.012 \\
\hline
\end{tabular}

Figure 18 shows the wind-speed-specific standard deviations for the yaw misalignment and upflow errors (Fig. 18a) and for the shear errors (Fig. 18b) as well as the wind Weibull distribution at FINO1 as functions of wind speed. The picture clearly illustrates the fact that for both angles and shears, errors are quite limited for the more probable wind speeds.

Finally, the lifetime standard deviations are reported in Table 2. From this point of view, the results are clearly quite satisfactory not only for shears, but also for angles. In fact, although fluctuations pollute the instantaneous observation of these quantities, their long-term metrics are well captured.

\subsubsection{Following mean changes in yaw misalignment}

The fact that the mean estimation errors of the angles, especially for yaw misalignment, are limited suggests the use of a moving average in order to lower the error standard deviation. This way one may capture the slower variations in the means while filtering out the faster oscillations. The resulting estimates can be used for slower control actions, for example yaw control, or for the slow scale monitoring of parameters of interest.

To test whether it is indeed possible to follow changes in the mean, large changes in yaw misalignment were simulated. Turbulent wind fields were generated with TurbSim and gradually rotated to generate mean wind direction changes from -4 to $4^{\circ}$ in about $20 \mathrm{~s}$. The observed yaw misalignment was filtered with a moving average of variable window length on account of the mean wind speed. The results of the observations at $7 \mathrm{~m} \mathrm{~s}^{-1}$ for different turbulence levels with and without moving average are shown in Fig. 19.

For the very low TI levels shown in Fig. 19a, both the mean and instantaneous values of yaw misalignment can be sufficiently well captured even without the use of a filter. With increasing turbulence, spurious oscillations of the estimates mask the mean wind direction change. However, it appears that the use of a moving average is capable of eliminating the faster fluctuations, revealing the presence of a change in wind direction. Clearly, higher values of turbulence require longer filtering windows with consequently longer time delays. This delayed detection is, however, compatible with the usually rather slow and conservative approach used for yaw control in which the actual realignment of the machine is performed only when a wind direction change of some significant entity has been observed for a sufficiently long window of time, usually many tens of seconds.

As a final remark, the nonlinear observer appears to perform slightly better than the linear one, as it is more easily visible for low turbulence conditions.

\section{Conclusions}

This paper has presented a method to estimate the wind inflow at the rotor disk of an operating wind turbine. The proposed method uses the low-frequency response of the wind turbine limited to the $1 \times \operatorname{Rev}$ harmonics to infer four wind states representing two misalignment angles and two shears. The rotor response is measured by load sensors, which are becoming standard equipment on many modern wind turbines. When such sensors are available, the proposed method does not require any additional hardware and amounts to a simple software upgrade. 

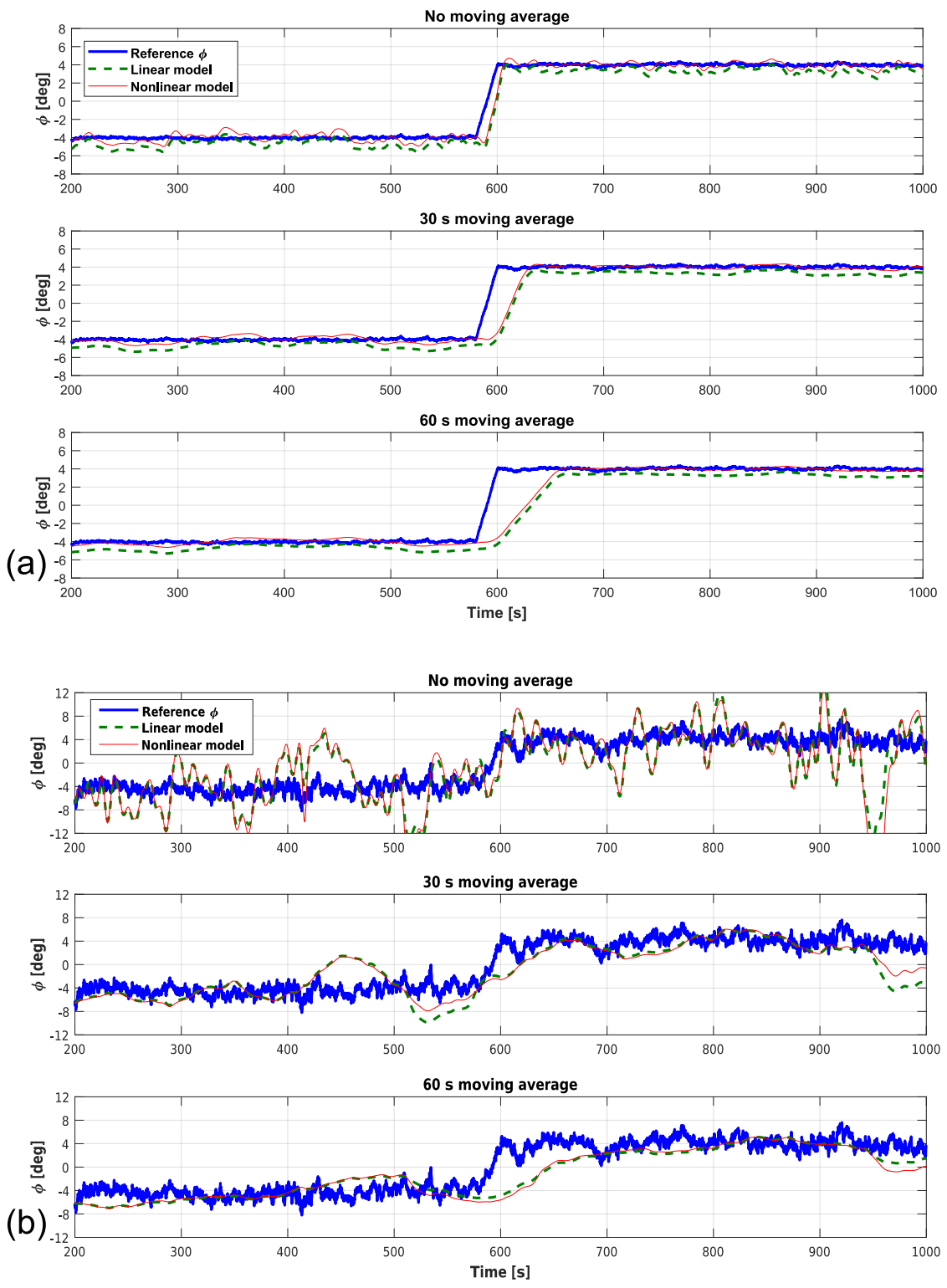

Figure 19. Yaw misalignment for an $8^{\circ}$ change in wind direction at $7 \mathrm{~m} \mathrm{~s}^{-1}$ with $2 \%$ (a) and $20 \%$ (b) TI. Solid thick blue lines: real yaw misalignment; dashed thick green lines: estimate by the linear model; solid thin red lines: estimate by the nonlinear model.

An input-output model was formulated to represent the relationship between wind states and load harmonics. The model was treated as a black box with unknown coefficients estimated by using the simulated response of a wind turbine implemented in a high-fidelity aeroservoelastic model. The input-output relationship was then inverted in a least-squares sense in order to provide estimates of the wind states when fed with measured load harmonics. The statistical properties of the model and, in turn, the observability of the wind states were analyzed using the SVD. This a priori analysis highlighted the different nature of the problem of estimating shears and angles, the former being characterized by a higher level of observability than the latter. Finally, the proposed ob- server was analyzed in a wide range of operating conditions in turbulent wind fields with different characteristics.

From the results of the present study, the following conclusions can be made.

- The behavior of the blade out-of-plane and in-plane load harmonics at $1 \times \operatorname{Rev}$ are captured well in both steady and turbulent conditions by a linear or second-order nonlinear function of the wind states.

- It is not advisable to include in the model harmonics that are higher than $1 \times$ Rev. In fact, although $2 \times$ Rev components are indeed correlated with wind states, they are also strongly affected by turbulence. In addition, if one 
uses a simulation model for the estimation or synthesis of the load-wind model, it is expected that such a model will better capture the $1 \times$ Rev response than the higher harmonics. Therefore, limiting load inputs to the $1 \times$ Rev components helps ensure higher accuracy of the load-wind model and hence of the estimates.

- Wind states can be estimated from $1 \times$ Rev blade harmonics, as these quantities carry enough informational content for the model to be invertible.

- An a priori observability analysis shows that the accuracy of the shears is generally superior to that of the angles. This is not because of a limit to the present specific formulation, but it is due to the intrinsic sensitivity of angle of attack changes to wind state changes, which is different for angles and shears.

- Extensive simulations in turbulent conditions have shown that the mean value of the estimation error is generally significantly low for all states. For example, the mean yaw error is about $0.5^{\circ}$ independently of wind speed and TI, whereas the vertical shear error is about 0.01 .

- Standard deviations of the shears are generally very low even for high TI levels, implying that the observer is capable of following fast shear fluctuations with good precision.

- Standard deviations for angles are significantly higher due to their overall lower observability. In general, angle estimates are polluted by rapid spurious oscillations due to the amplification of errors through the inverted estimation model. This implies that one cannot generally follow rapid variations in the angles, and only observations on longer timescales are possible.
- Although polluted by fluctuations, on average even the angle estimates are of good quality thanks to their small mean errors

- An analysis, conducted by taking into account the probability distributions of both wind speed and TI at the offshore FINO1 platform in the German Bight, has shown that the expected standard deviation of the estimation error in the angles is below $1^{\circ}$, which appears to be a very interesting result.

- It was shown that, by filtering the estimated yaw misalignment with a moving average, one may track with good accuracy significant mean changes in the wind direction even for very high $\mathrm{TI}$, indicating the possible use of this estimate to drive the wind turbine yaw control system.

The proposed formulation should be extended to consider the possible presence of an individual pitch control (IPC) strategy. This can be done by also including in the loadwind model the presence of a term depending on pitch load harmonics. As these quantities are known, they represent further inputs that do not change the overall approach, although the model will have additional coefficients that need to be identified. This extension of the formulation has already been tested, and it will be described in a forthcoming publication.

Data availability. Data can be obtained upon request from the authors (carlo.bottasso@tum.de.). 
Appendix: Nomenclature

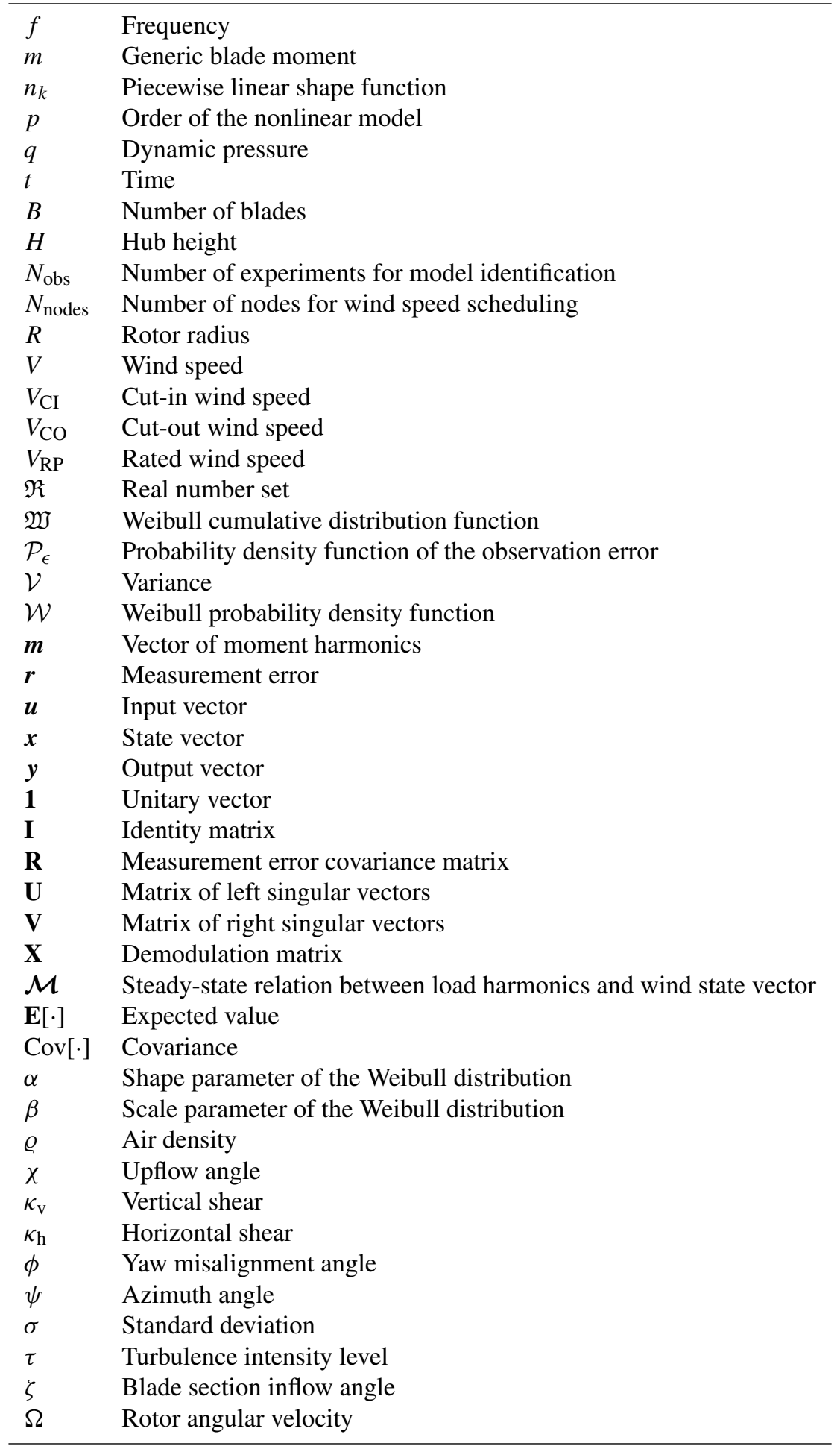




$\begin{array}{ll}\boldsymbol{\epsilon}_{\theta} & \text { Wind state observation error } \\ \boldsymbol{\xi} & \text { Vector of statistically independent wind state variables, } \mathbf{V}^{T} \boldsymbol{\theta} \\ \boldsymbol{\theta} & \text { Wind state vector } \\ \boldsymbol{\Sigma} & \text { Rectangular matrix of singular values } \\ (\cdot) & \text { Time derivative, i.e., } \cdot \cdot / \mathrm{d} t \\ (\cdot)^{(i)} & \text { Quantity related to the } i \text { th experiment } \\ (\cdot)_{(j)} & \text { Quantity related to the } j \text { th blade } \\ (\cdot)_{k} & \text { Nodal quantity at the } k \text { th node } \\ (\cdot)_{n \mathrm{c}} & n \times \text { Rev cosine amplitude } \\ (\cdot)_{n \mathrm{~s}} & n \times \text { Rev sine amplitude } \\ (\cdot)_{\mathrm{E}} & \text { Estimated quantity } \\ (\cdot)^{\mathrm{IP}} & \text { In-plane quantity } \\ (\cdot)_{\mathrm{M}} & \text { Measured quantity } \\ (\cdot)_{\mathrm{NL}} & \text { Nonlinear term } \\ (\cdot)^{\mathrm{OP}} & \text { Out-of-plane quantity } \\ (\cdot)_{\mathrm{R}} & \text { Real quantity } \\ (\cdot)^{\mathrm{T}} & \text { Transpose } \\ \mathrm{BEM} & \text { Blade element momentum } \\ \mathrm{CDF} & \text { Cumulative distribution function } \\ \mathrm{IPC} & \text { Individual pitch control } \\ \text { Lidar } & \text { Light detection and ranging } \\ \mathrm{PDF} & \text { Probability density function } \\ \text { SD } & \text { Standard deviation } \\ \text { SVD } & \text { Singular value decomposition } \\ \mathrm{TI} & \text { Turbulence intensity } \\ \mathrm{TSR} & \text { Tip speed ratio } \\ & \end{array}$


Competing interests. The authors declare that they have no conflict of interest.

This work was supported by the German Research Foundation (DFG) and the Technische Universität München within the funding program

Open Access Publishing.

Edited by: Sandrine Aubrun

Reviewed by: two anonymous referees

\section{References}

Bauchau, O. A.: Flexible Multibody Dynamics, Solid Mechanics and its Applications, Springer, Dordrecht, Heidelberg, London, New York, 2011.

Bauchau, O. A., Bottasso, C. L., and Trainelli, L.: Robust integration schemes for flexible multibody systems, Comput. Method. Appl. M., 192, 395-420, https://doi.org/10.1016/S00457825(02)00519-4, 2003.

Bossanyi, E., Fleming, P. A., and Wright, A. D.: Controller field tests on the NREL CART3 turbine, Technical Report 11593/BR/09, National Renewable Energy Laboratory, Golden, CO, USA, 2009.

Bottasso, C. L. and Croce, A.: Cp-Lambda: user's manual, Technical Report, Dipartimento di Ingegneria Aerospaziale, Politecnico di Milano, Milano, Italy, 2006.

Bottasso, C. L. and Riboldi, C. E. D.: Estimation of wind misalignment and vertical shear from blade loads, Renew. Energ., 62, 293-302, https://doi.org/10.1016/j.renene.2013.07.021, 2014.

Bottasso, C. L. and Riboldi, C. E. D.: Validation of a wind misalignment observer using field test data, Renew. Energ., 74, 298-306, https://doi.org/10.1016/j.renene.2014.07.048, 2015.

Bottasso, C. L., Croce, A., and Riboldi, C. E. D.: Spatial estimation of wind states from the aeroelastic response of a wind turbine, The Science of Making Torque from Wind (TORQUE 2010), Heraklion, Crete, Greece, 28-30 June 2010, 2010.

Bottasso, C. L., Croce, A., Nam, Y., and Riboldi, C. E. D.: Power curve tracking in the presence of a tip speed constraint, Renew. Energ., 40, 1-12, https://doi.org/10.1016/j.renene.2011.07.045, 2012.

Bottasso, C. L., Cacciola, S., and Schreiber, J.: A wake detector for wind farm control, J. Phys. Conf. Ser., 625, 012007-1-8, https://doi.org/10.1088/1742-6596/625/1/012007, 2015.

Bottasso, C. L., Cacciola, S., and Schreiber, J.: Local wind speed estimation, with application to wake impingement detection, Renew. Energ., 116, 155-168, https://doi.org/10.1016/j.renene.2017.09.044, 2018.

Cacciola, S., Bertelè, M., Schreiber, J. and Bottasso, C. L.: Wake center position tracking using downstream wind turbine hub loads, J. Phys. Conf. Ser., 753, 032036-1-6, https://doi.org/10.1088/1742-6596/753/3/032036, 2016a.

Cacciola, S., Bertelè, M., and Bottasso, C. L.: Simultaneous observation of wind shears and misalignments from rotor loads, J. Phys. Conf. Ser., 753, 052002-1-8, https://doi.org/10.1088/1742-6596/753/5/052002, 2016b.

Coleman, R. P. and Feingold, A. M.: Theory of self-excited mechanical oscillations of helicopter rotors with hinged blades, Technical Report, NACA TN 1351, 1958.
Cramér, H.: Mathematical Methods of Statistics, Princeton University Press, Princeton, NJ, USA, 1946.

Eggleston, D. M. and Stoddard, F. S.: Wind Turbine Engineering Design, Van Nostrand Reinhold, New York, USA, 1987.

Emeis, S.: Wind Energy Meteorology - Atmospheric Physics for Wind Power Generation, Springer-Verlag, Berlin, Heidelberg, Germany, 2013.

FINO: FINO 1-2-3 - Forschungsplattformen in Nord- und Ostsee Nr. 1, available at: http://www.fino1.de/en/, last access: 13 November 2017.

Fleming, P. A., Wright, A. D., Fingersh, L. J., and van Wingerden, J. W.: Resonant vibrations resulting from the re-engineering of a constant-speed 2-bladed turbine to a variable-speed 3-bladed turbine, Proceedings of the 49th AIAA Aerospace Sciences Meeting, Orlando, FL, USA, 2011.

Fleming, P. A., Gebraad, P. M. O., Lee, S., van Wingerden, J. W., Johnson, K., Churcheld, M., Michalakes, J., Spalart, P., and Moriarty, P.: Evaluating techniques for redirecting turbine wakes using SOWFA, Renew. Energ., 70, 211-218, https://doi.org/10.1016/j.renene.2014.02.015, 2014.

Golub, G. H. and van Loan, C. F.: Matrix Computations, Johns Hopkins University Press, Baltimore, MD, USA, 1996.

Jategaonkar, R. V.: Flight Vehicle System Identification: a Time Domain Methodology, AIAA, Progress in Astronautics and Aeronautics, Reston, VA, USA, 2nd edn., 2015.

Jimenez, A., Crespo, A., and Migoya, E.: Application of a LES technique to characterize the wake detection of a wind turbine in yaw, Wind Energy, 13, 559-572, https://doi.org/10.1002/we.380, 2010.

Jonkman, B. J. and Kilcher, L.: TurbSim user's guide: version 1.06.00, NREL Technical report, 2012.

Ljung, L.: Perspectives on system identification, Annu. Rev. Control, 34, 1-12, 2010.

Moré, J. J.: The Levenberg-Marquardt algorithm: implementation and theory, Numerical Analysis: Proceedings of the Biennial Conference Held at Dundee, 28 June-1 July 1977, Springer, Berlin Heidelberg, Germany, Lect. Notes Math., 630, 105-116, https://doi.org/10.1007/BFb0067700, 1978.

Riboldi, C. E. D.: Advanced control laws for variable-speed wind turbines and supporting enabling technologies, $\mathrm{PhD}$ thesis, $\mathrm{Po-}$ litecnico di Milano, Milano, Italy, 2012.

Simley, E. and Pao, L. Y.: Evaluation of a wind speed estimator for effective hub-height and shear components, Wind Energy, 19, 167-184, https://doi.org/10.1002/we.1817, 2014.

Soltani, M. N., Knudsen, T., Svenstrup, M., Wisniewski, R., Brath, P., Ortega, R., and Johnson, K.: Estimation of rotor effective wind speed: a comparison, IEEE T. Contr. Syst. T., 21, 1155-1167, https://doi.org/10.1109/TCST.2013.2260751, 2013.

Türk, M. and Eimeis, S.: The dependence of offshore turbulence intensity on wind speed, J. Wind Eng. Ind. Aerod., 98, 466-471, https://doi.org/10.1016/j.jweia.2010.02.005, 2010.

Van der Hooft, E. L. and Engelen, T.: Estimated wind speed feed forward control for wind turbine operation optimisation, European Wind Energy Conference \& Exhibition (EWEC 2004), London, UK, 22-25 November 2004. 